Investigación Valdizana, vol. 15, núm. 2, 2021, pp. 89-100.

\title{
El arborigrama. Estrategia didáctica de comprensión lectora de textos narrativos.
}

\author{
Loayza-Maturrano, Edward Faustino.
}

Cita:

Loayza-Maturrano, Edward Faustino (2021). El arborigrama. Estrategia didáctica de comprensión lectora de textos narrativos. Investigación Valdizana, 15 (2), 89-100.

Dirección estable: https://www.aacademica.org/edward.faustino.loayza.maturrano/19 ARK: https://n2t.net/ark:/13683/p8Mo/3k2

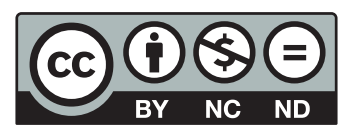

https://creativecommons.org/licenses/by-nc-nd/4.0/deed.es.

Acta Académica es un proyecto académico sin fines de lucro enmarcado en la iniciativa de acceso abierto. Acta Académica fue creado para facilitar a investigadores de todo el mundo el compartir su producción académica. Para crear un perfil gratuitamente o acceder a otros trabajos visite: https://www.aacademica.org. 


\title{
El arborigrama. Estrategia didáctica de comprensión lectora de textos narrativos
}

\section{The Arborigram. Didactic strategy of reading comprehension of narrative texts}

\author{
Edward F. Loayza Maturrano ${ }^{1, a, *}$
}

\begin{abstract}
Resumen
El bajo nivel de comprensión de lectura evidenciado por los estudiantes de la educación básica exige plantear propuestas que vinculen de forma eficaz dos variables socioeducativas: la didáctica y la lectura. La investigación planteó como objetivo principal determinar si existe influencia entre la estrategia del arborigrama y la mejora de la comprensión lectora en una población pre-adolescente del grupo etario de 11 a 13 años del primer grado del nivel Secundaria en el distrito de La Molina. Se diseñó un estudio cuasiexperimental que consideró un pre-prueba y post-prueba respecto de la aplicación del módulo del arborigrama. Los resultados indican la existencia de influencia causal moderada alta entre las variables estrategia del arborigrama y la comprensión de lectura en los estudiantes. La conclusión principal del estudio establece que el arborigrama como estrategia didáctica influye de forma significativa en la mejora de la comprensión de lectura de textos narrativos en estudiantes de la educación básica.
\end{abstract}

Palabras clave: estrategias de lectura, comprensión lectora, estrategias cognitivas, mejora de la lectura, logro de lectura.

\begin{abstract}
The low level of reading comprehension evidenced by basic education students requires proposing proposals that effectively link two socio-educational variables: didactics and reading. The main objective of the research was to determine if there is influence between the arborigram strategy and the improvement of reading comprehension in a pre-adolescent population of the age group of 11 to 13 years of the first grade of the Secondary level in the district of La Molina. A quasi-experimental study was designed that considered a pre-test and post-test regarding the application of the arborigram module. The results indicate the existence of a moderate-high causal influence between the variables of the arborigram strategy and the students' reading comprehension. The main conclusion of the study establishes that the arborigram as a didactic strategy has a significant influence on the improvement of reading comprehension of narrative texts in students of basic education.
\end{abstract}

Keywords: reading strategies, reading comprehension, cognitive strategies, reading improvement, reading achievement.

${ }^{1}$ Universidad Nacional Agraria La Molina, Perú

E-mail, aedwloma@lamolina.edu.pe

Orcid ID: "https://orcid.org/0000-0002-1359-8414 
Introducción

Si hiciéramos un recordaris de nuestras primeras experiencias con el texto, en la empresa de comprender lo que se va leyendo, diríamos que uno recurría a ciertas estrategias visuales como dibujos y textos; o sea, a una especie de sumario en la hoja de papel de lo destacado, de lo que más nos interesó, en suma, de lo comprendido. En consecuencia, esta acción de representar, de sintetizar, de registrar lo que se entendió; supone una práctica de esquematización casi por intuición. Ningún adulto escolarizado pudo haber hecho su recorrido académico sin esbozar algún tipo de esquema: un diagrama, cuadro, tabla o figura que resumiera el texto leído. Entonces, el recurrir a este recurso denominado en términos generales como "el esquema" es, qué duda cabe, una necesidad imprescindible en el afán de comprender un texto.

A lo largo de la historia, las prácticas de lectura han ido variando considerablemente. Desde una mera traducción oral en voz alta; esto es, una lectura para ser escuchada a otra silenciosa y de estudio; y finalmente, a otra actual: la denominada lectura digital. Esto ha hecho que los modos de interpretar los significados de los textos también se hayan transformado en función de los distintos sistemas de pensamiento, distintos sistemas culturales; los cuales se han ido reeditando con el paso del tiempo. La lectura ha transitado del fuero de lo privado y privativo al ámbito de lo público y popular. De este modo, ha devenido en uno de principales medios para aprender y comunicar, y se constituye insoslayablemente en una herramienta eficaz para comprender la cultura. Por todo lo anterior, las estrategias de lectura también han ido cambiando según las nuevas exigencias e intereses; se han ido replanteando a través del tiempo. Así, la organización visual de las ideas del texto; o sea, el empleo de organizadores gráficos de la información en las prácticas lectoras se erige como una de las estrategias más eficaces por su significancia y utilidad para comprender un texto (García Madruga, 2006; Gonzales, 2017).

En tal sentido, Duke \& Pearson (2002) refiriéndose a este punto sobre "la representación visual del texto" como estrategia de lectura explica que justamente son eso re-presentaciones, esto es, una nueva presentación original de lo leído, pero que pese a ser una transformación activa del contenido, lo refleja fielmente. Aquí entra en juego significativo y sinérgico los conocimientos previos, la percepción, la memoria y los procesos cognitivos de la comprensión; todo lo cual en una interacción virtuosa. De este modo, toda estrategia que conduzca nuevamente al lector al texto en un lenguaje cercano al original, facilitará su conocimiento, su recuperación, su conexión con otros textos; en suma, encaminará su comprensión. En un estudio de revisión sistemática sobre estrategias de comprensión de lectura realizado por Pearson \& Fielding (1991, pp. 828 y ss.) se identifica algo más de una docena de variantes en la categoría de estrategias basadas en representaciones visuales de las ideas de un texto, entre las que destacan: el mapeo o los mapas sobre el texto, los diagramas de flujo, el trabajo en redes o el networking y los organizadores gráficos (Bean, Singer, Sorter \& Frazee, 1986). Los resultados de estas investigaciones comprueban cuatro hallazgos que estas representaciones esquemáticas del texto facilitan: 1) la memorización; 2) la habilidad de respuesta en la resolución de preguntas; 3) la sumarización o resumen $y$; 4) la comprensión global del texto. Otros estudios y revisiones sistemáticas más recientes comprueban la capacidad que tienen los organizadores visuales de la información del texto en el desarrollo de competencias lectoras de los estudiantes (Kim, Vaughn, Wanzek \& Wei, 2004; Manoli \& Papadopoulou, 2012; Thayne, 2018).

Si bien muchos de los problemas de la vida diaria se pueden resolver buscando una dirección, rellenando un formulario y toda forma de alcanzar un cierto grado de saber comprensivo; en el ámbito educativo, con mayor razón la comprensión lectora es imprescindible y fundamental, pues permite que el estudiante logre otros aprendizajes y vaya escalando en nivel de complejidad sus interpretaciones, logrando comprender en el plano intertextual, macrotextual e hipertextual. Por tanto, la comprensión lectora es una capacidad compleja ya que interactúan procesos perceptivos, cognitivos, lingüísticos y socioculturales; y todos juntos logran la comprensión cabal del texto. Hasta aquí los diversos enfoques e investigaciones referidas demuestran que en la comprensión lectora se implican un conjunto de habilidades y destrezas más que un cuerpo de conocimientos sobre hechos o conceptos. Estas habilidades se adquieren con el ejercicio lector continuo y con el uso de métodos, técnicas y estrategias de lectura (Dubois, 1984; Izquierdo \& Rodríguez, 2020).

Otro aspecto central en el estudio de la lectura es el modo de concebirla. Los paradigmas epistemológicos sobre la lectura que más aceptación e influencia han conseguido son el positivismo y el constructivismo; los cuales también han tenido un mayor impacto en su investigación y desarrollo científico. Esta cientificidad ha generado enfoques sociológicos, psicolingüísticos y psicológicos (Guerra, 2017). De este modo, estos enfoques han dado lugar a modelos tales como: el conductismo, el cognitivismo, el interaccionismo y el constructivismo. En cada uno de estos modelos subyace una concepción distinta de lector, del texto y de su contexto. En el conductismo el lector es concebido como un sujeto que sigue las reglas, las pautas y aprende a leer por repetición y ejercitación de dichas normas y ejercicios; el texto es visto como tarea y su contexto es la resolución de baterías de preguntas (Paris, Cross \& Lipson, 1984; Cain \& Oakhill, 2014). 
El cognitivismo comprende un lector como un sistema complejo mental que se encarga del procesamiento de la información donde existe un input (los datos percibidos mediante el proceso de transducción sensorial) y un output (desciframiento de la información: la comprensión textual por intercurrencia cerebral); el texto es concebido como datos y su contexto son todas las funciones cognitivas que entran en juego tanto en la construcción del texto (por el autor) como en el desciframiento (por el lector): la percepción, la codificación lingüística, la decodificación lingüística, la memoria, la categorización y el razonamiento. Un modelo que tuvo escasa resonancia en la educación sobre la lectura, pero que permitió identificar las distintas funciones cognitivas involucradas en la lectura.

El interaccionismo concibe al lector como un elemento activo y fundamental en la lectura. Existe un espacio de interacción pragmática entre el que lee y el escritor, que le da nuevos sentidos al texto; es decir, un contexto: una situación comunicativa que resignifica. El valor del texto radica en esta comunicación significativa que efectúa el lector. Por su parte, el constructivismo comprende al lector como ser reflexivo y agente cognitivo en el proceso comprensor, el cual está vinculado estrechamente con el plano sociocultural; razón por el que se configura en un enfoque sociocognitivo (Solé y Teberosky, 2001). La metacognición se convierte en la principal acción para lograr la comprensión lectora significativa del texto/discurso (Flavell 1984; Short \& Ryan 1984; Ehrlich, 1999; Coll, 2001). Se considera la teoría Vygotskyana de las zonas de desarrollo (zonas de desarrollo real, de desarrollo próximo y de desarrollo potencial) y la teoría del análisis crítico del discurso (ACD) de Van Dijk y Kinstch. De este modo, se concibe al texto vinculado al contexto y la cultura. La responsabilidad principal de la comprensión de lectura recae en el lector quien tiene que emplear estrategias eficaces según el tipo del texto y los propósitos lectores predefinidos (Dijk \& Kinstch, 1983; Solé, 1996; Solé y Teberosky, 2001).

En este sentido, en la actualidad se disponen de una diversidad de estrategias cognitivas que facilitan la comprensión de lectura. Unas estrategias son más globales y multidimensionales como las planteadas por Kenneth Goodman y otras más específicas y funcionales como, por ejemplo, las desarrolladas en las investigaciones aplicadas de Susana Pérez y José Villalobos. Las estrategias cognitivas generales que posibilitan la comprensión lectora son: 1). La estrategia de iniciación que supone el registro mental de algo pasible de ser leído (texto leíble) por parte del lector a través de la observación visual que realiza del entorno, por ejemplo, la portada o el título de un libro. Esta estrategia significa una toma de decisión deliberada sobre el acto de la lectura. 2). Las estrategias combinadas del muestreo y selección que refieren, en primer momento, a los saltos de vista que se realiza el lector según aquello sea de su mayor interés o bien si la información le es más productiva o útil; $y$, en segundo momento, a la eficiencia en la selectividad de los datos más relevantes. 3). La estrategia de la inferencia consiste en las deducciones que efectúa el lector empleando su campo de experiencias y los saberes previos con que cuenta; luego conforme avance en la lectura se irán explicitando o no dichas inferencias, y conforme algunas de estas inferencias las vaya validando o no, irá incrementando su nivel de confianza y comprensión lectora. 4). La estrategia de predicción consiste en la capacidad/acción del lector de anticipar y predecir lo que seguirá en la lectura, para lo cual emplea tanto la información explícita como la inferida. 5). La estrategia de confirmación permite al lector ir determinando la congruencia entre la comprensión textual previa, las predicciones e inferencias y la información nueva; por lo que se irá validando lo predicho o lo anticipado. 6). La estrategia de corrección que consiste en volver a leer el texto para recopilar más información, y luego, volver a analizar la información leída con el fin de realizar interpretaciones, inferencias y predicciones alternativas; y de este modo, poder recuperar el significado correcto. 7). La estrategia de terminación se emplea de distintas maneras por parte del lector de acuerdo a razones de falta de interés, poca comprensión, escaso tiempo, aburrimiento, desidia o cuando existe variación en las circunstancias; por lo que no se aplica solo en el momento en que se termina de leer un texto (Block, 1986; Goodman, 1996).

Las estrategias cognitivas específicas y funcionales que permiten la comprensión lectora son: 1). La estrategia del cuestionamiento en la que el lector realiza preguntas a sí mismo, preguntas al autor/texto y, de manera puntual el cuestionar el significado de un enunciado o cláusula, también el cuestionar el significado de una palabra. 2). La estrategia del parafraseo oral (subvocalización/ vocalización) que consiste en el replanteamiento simplificado de la información del texto facilitando de este modo la comprensión del significado textual. Esta estrategia permite la simplificación y aclaración del contenido, por ejemplo, a través del uso de sinónimos o simplificando construcciones sintácticas redundantes. 3). La estrategia de la relectura de salteo que supone volver a leer ciertas partes del texto que posee mayor importancia para el lector o goza de mayor atención e interés según los propósitos de la lectura. 4). La estrategia del resumen que consiste en describir brevemente el contenido del texto leído en la mente o de forma oral. 5). La estrategia de asociación conceptual que refiere a la acción del lector cuando establece relaciones entre los conocimientos previos y la información nueva, o cuando se determinan las relaciones entre los nuevos conceptos y los ya aprendidos. 6). La estrategia de detección de coherencia que consiste en descubrir las segmentaciones temáticas, las secuencias 
textuales dominantes aplicando criterios de inclusión y exclusión; así como al identificar la organización lógica de la información clave, o al identificar las lógicas de producción textual y las ideas de desarrollo. 7). La estrategia de monitoreo que supone dar seguimiento respecto de las fortalezas y debilidades en la comprensión textual; así como en la identificación de conceptos erróneos en la lectura. 8). La estrategia mnemotécnica que consiste en acciones para recordar detalles de información relevante, por ejemplo, mediante el encadenamiento de conceptos en una oración, o a través del empleo de una ruta espacial conocida en la que se va colocando visualmente cada elemento conceptual en una ubicación particular a lo largo de la ruta (Derry \& Murphy, 1986; Sarig, 1987; Paris, Wasik \& Turner, 1991; Pérez, 2015; Villalobos, 2020).

En tal sentido, las estrategias didácticas de comprensión de lectura asumen estas estrategias cognitivas como basamento teórico-procedimental para generar intervenciones válidas que posibiliten la comprensión textual. Así por ejemplo, al emplear un mapa conceptual como estrategia esquemática gráfica se refuerzan algunas estrategias cognitivas multidimensionales como las de muestreo y selección, y las estrategias cognitivas específicas de detección de coherencia, la de asociación conceptual y la de resumen (Meyer, 1984; Scardamalia \& Bereiter, 1984; Pérez, 2015).

La estrategia del arborigrama se constituye en un organizador gráfico de la información que se sustenta en las estrategias cognitivas que facilitan la comprensión de lectura, cuya estructura lógica permite muestrear, seleccionar, parafrasear, sintetizar información relevante sobre lo leído. Asimismo, el arborigrama se perfila como una estrategia ludo-iconoglósica, ya que permite que el estudiante rediseñe, coloree y use también, en general, su creatividad en el proceso de comprensión lectora. Específicamente, esta estrategia consiste en organizar esquemáticamente un texto narrativo o un tópico de aprendizaje dentro de la iconocidad del árbol, donde se reconocen sus partes: raíces, tronco, ramas, hojas, flores y frutos, y análogamente se le va asignando los temas, subtemas, ideas fuerza, propuestas a cada componente del árbol respectivamente. Está considerada dentro de las estrategias de aprendizaje para el procesamiento de la información (Loayza, 2020).

De este modo, permite reconocer de manera panorámica y en conjunto el contenido a aprender. $Y$ al apreciar el esquema completo del tema de estudio, posibilita la elaboración de clasificaciones, categorizaciones que ayudarán a alcanzar una comprensión integral del tema abordado (Mc Daniel \& Einstein, 1991). Para aplicar adecuadamente la estrategia del arborigrama el maestro a través de casos desarrollados (arborigramas resueltos) mediará el aprendizaje de los estudiantes (Stull \& Mayer, 2007). Posteriormente, los discentes harán presentaciones de sus propios arborigramas en hojas pequeñas de papel o papelotes. En este proceso se comprobará el nivel de comprensión

Figura 1. Arborigrama aplicado al relato El gato negro de Edgar Poe (enfoque temático)

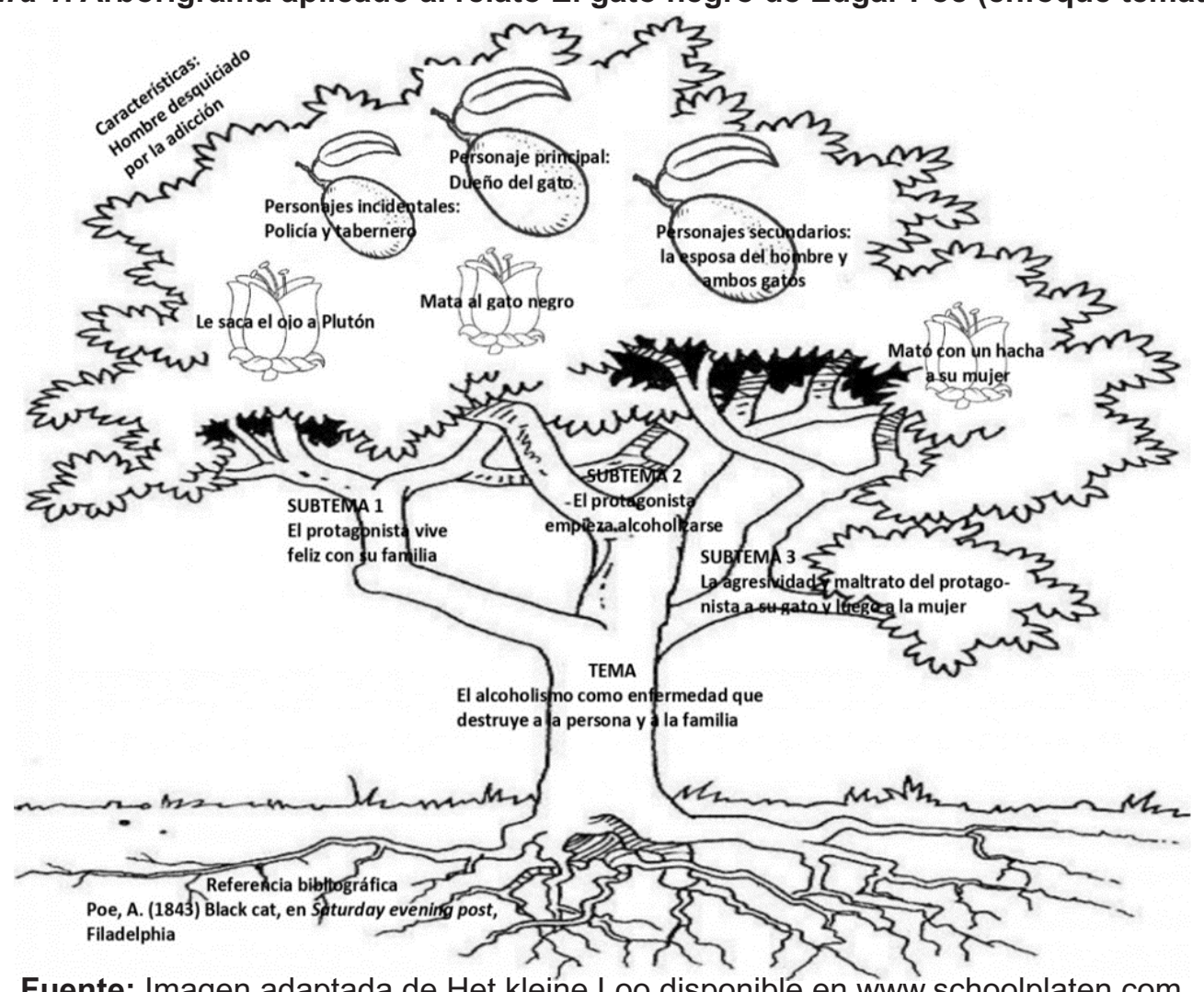

Fuente: Imagen adaptada de Het kleine Loo disponible en www.schoolplaten.com 
que alcanzan los estudiantes según los criterios de contenido (Levin \& Pressley, 1983; Pressley, 1999). Para tal efecto, el estudiante o el equipo de estudio debe releer, contrastar y analizar el tema y seguidamente organizarlo todo en el esquema de árbol, reconociendo que en el tronco del árbol se coloca el tema a aprender, en cada rama van los subtemas, en las hojas se anotan las características principales, en las flores se muestran las evidencias o ejemplos, en los frutos se escriben informaciones adicionales relevantes; y en las raíces se anotan las referencias o fuentes (Loayza, 2020; Prado-Huarcaya y Escalante-López, 2020).

Las esquematizaciones del arborigrama, asimismo, permite ser una estrategia aplicable tanto durante el desarrollo de una sesión de aprendizaje o como actividad de extensión de los aprendizajes, es decir, convertirse en una actividad de reforzamiento de saberes o como tarea complementaria en otra área curriculares como en las áreas de Ciencias Sociales e Idioma Extranjero. Por otro lado, si bien es posible aplicarlo en el procesamiento de la información de textos expositivos como lo señalado anteriormente, también es aplicable a textos narrativos; con lo cual se ampliaría el espectro de funcionalidad de la estrategia en la didáctica de la comprensión lectora (Stull \& Mayer, 2007).

Una aplicación de esta de estrategia didáctica de comprensión lectora se muestra en la figura 1.

El propósito de esta estrategia es que el estudiante desarrolle su aprendizaje mediante la transposición de sus relaciones cognitivas sobre lo que lee en un esquema gráfico que materialice dichas relaciones conceptuales. De este modo, se refuerzan distintas estrategias cognitivas generales como las de muestreo y selección, de inferencia, de predicción, confirmación y corrección. Igualmente, se consolidan las estrategias cognitivas específicas como las de cuestionamiento, de parafraseo, de relectura, de resumen y de detección de coherencia principalmente. En ese sentido, una aplicación de detalle de la estrategia es la que permite la identificación del hecho principal en el tronco, los hechos secundarios en las raíces, el tiempo de la historia en las hojas, la identificación de personajes en los frutos, en las flores los valores del relato y sobre la copa del árbol el problema de la historia (Novak \& Cañas, 2008).

La representación icónica del árbol (fig. 2) supone una representación visual de la información que permite una fácil captación perceptual de parte de los estudiantes. En ese sentido, expresar lo comprendido a través de un esquema visual favorece la retención de los conceptos e ideas relevantes de lo leído, esta condición es corroborada con estudios anteriores (Thorndyke \& HayesRoth, 1979; Düsrteler, 2002; Novak \& Cañas, 2008). Asimismo, el arborigrama como esquema gráfico que refuerza las estrategias cognitivas que facilitan la comprensión de lectura como la selección y memorización de la información relevante, por lo que de forma didáctica se contribuye a ampliar el conocimiento del texto; todo lo cual se afianza con los hallazgos de estudios experimentales más actuales efectuados con

Figura 2. Arborigrama aplicado al relato El gato negro de Edgar Poe (enfoque de eventos)

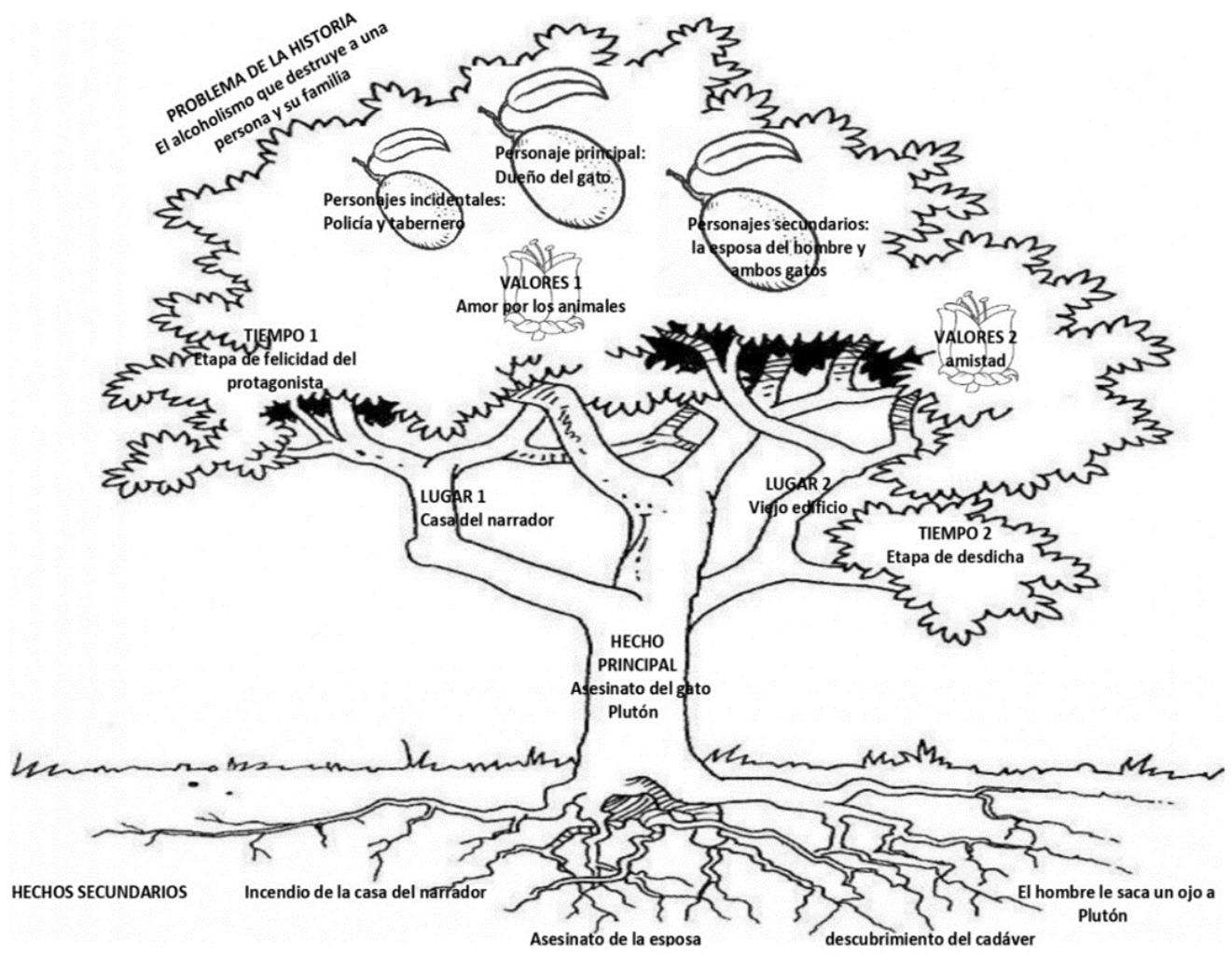


estudiantes, en el que se determina que los organizadores gráficos pre-elaborados facilitados por el docente, como los planteados en el módulo de la estrategia del arborigrama de la presente investigación mejoran la retención, la categorización y la comprensión de lectura (Guerra, 2017; Colliot \& Jamet, 2018).

\section{Metodología}

El presente estudio es una investigación cuantitativa y de nivel aplicado porque se establece mediante un análisis contable y empleando números reales la influencia de una estrategia. Asimismo, es de tipo transversal debido a que se hará un corte en el tiempo; esto es, en un solo momento, de forma sincrónica.

\section{Diseño}

El diseño de la investigación es cuasiexperimental, debido a que el estudio consiste en determinar el grado de influencia que provoca la variable independiente (estrategia del arborigrama) respecto de la variable dependiente (la comprensión de lectura).

\section{Participantes}

La población está constituida por 220 estudiantes del primer grado del nivel Secundaria de una Institución Educativa Pública en el distrito de La Molina. Los estudiantes estuvieron divididos en siete aulas diferenciadas por letras desde A hasta la $G$ cada una con un promedio de treinta estudiantes (+1 ó -1) entre varones y mujeres.

\section{Materiales}

La técnica considerada en la investigación es la prueba o test. Se aplicó el test de comprensión lectora (variable dependiente). El instrumento contó con veinte ítems de comprensión de lectura, el cual se empleó como prueba de pre y post test, cuyos contenidos están en relación con el módulo de la estrategia del arborigrama. Para la validez interna o confiabilidad del test, se realizó una prueba piloto con diez estudiantes, a quienes se les administró dicho cuestionario, luego se calificó las diez pruebas y se empleó el estadígrafo de fiabilidad de instrumento Kuder-Richardson 20 (KR-20) a fin de determinar su consistencia interna. El resultado indicó un coeficiente de 0,827 considerado como de alta confiabilidad, lo cual permitió la aplicación en la muestra de estudio.

\section{Procedimientos}

Los test se aplicaron en dos momentos diferentes a los mismos estudiantes (pre test, post test). Los participantes fueron informados previa a la aplicación de los test respecto del objetivo del estudio con la intención de que no se produzca ninguna distorsión de las respuestas. Luego de hacer el cálculo del muestreo, el resultado correspondió $n=30$ redondeado correspondiente al tamaño de la muestra de estudio. Así, el aula identificada como la sección $\mathrm{C}$ se consideró como grupo muestral de experimento y al aula de la sección D como grupo de control. Finalmente, obtenido los resultados de los test, se utilizó el programa estadístico SPSS-26 para los análisis descriptivos e inferenciales.

\section{Análisis de datos y discusión}

Los resultados obtenidos después del desarrollo de las sesiones de aprendizaje aplicando la estrategia del arborigrama se corroboran diferencias significativas en el grupo-experimento respecto del grupo-control. De este modo, la aplicación final de los post test permitió contar con los datos necesarios para realizar los análisis necesarios, los contrastes intragrupales e intergrupales (tabla 1).

Tabla 1

Estadísticas descriptivas de los grupos experimental y de control

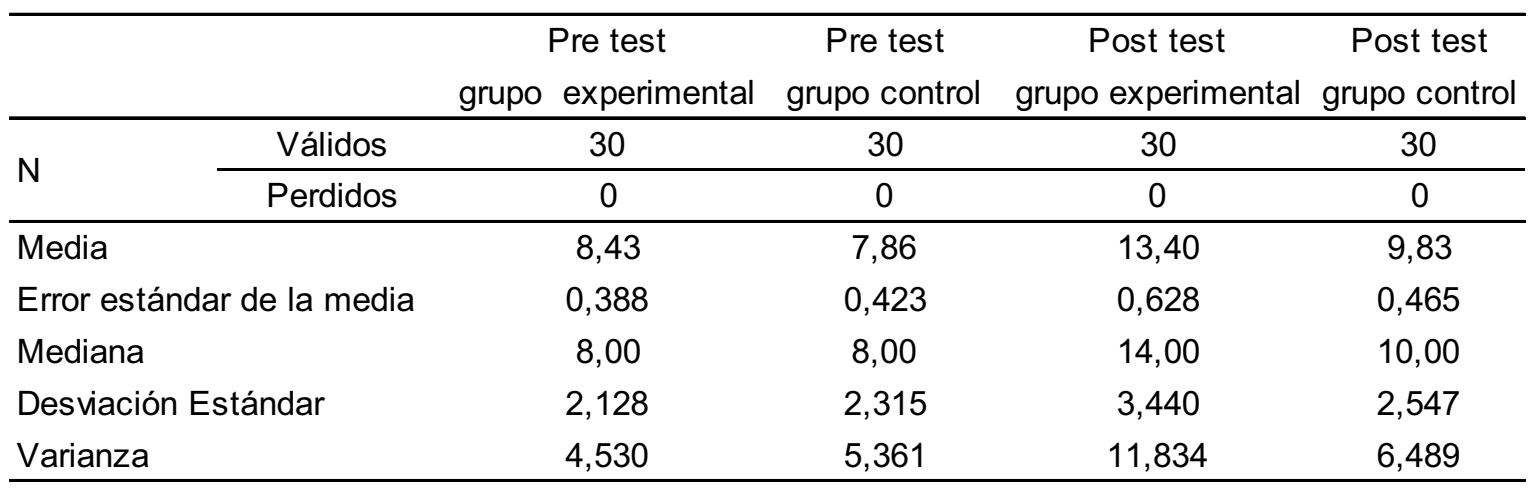

Fuente: Elaboración propia

Según lo observado en la Tabla 1 , la media aritmética del grupo de control es de 9,83 y del grupo experimental de 13,40 , los cuales evidencian diferencias significativas $(9,83 ; 13,40)$; resultando el grupo experimental con 3,57 puntos a su favor, además el promedio del grupo control es desaprobatorio y del grupo experimental aprobatorio normal, con varianzas y desviaciones estándar del grupo experimental de 11,834 y 3,440 frente al grupo control de 6,489 y 2,547 respectivamente, indicando que cada grupo es ligeramente homogéneo, pero entre grupos son heterogéneos. Descriptivamente, estos datos permiten afirmar que el desarrollo del módulo de la estrategia del 
arborigrama en la comprensión lectora en el grupo experimental obtuvo mejores resultados respecto del grupo de control al cual no se le aplicó la estrategia.

La hipótesis principal de investigación enuncia que la ejecución de la estrategia del arborigrama influye de forma significativa en la comprensión de lectura de los estudiantes del primer grado del nivel
Secundaria de las Instituciones Educativas Públicas en el distrito de La Molina. Esta hipótesis permite generar la hipótesis nula específica a falsar: La aplicación de la estrategia del arborigrama no influye de forma significativa en la comprensión lectora de los estudiantes del primer grado del nivel Secundaria de las Instituciones Educativas Públicas en el distrito de La Molina.

Tabla 2

Prueba de hipótesis general de la investigación

\begin{tabular}{|c|c|c|c|c|c|c|c|c|c|c|}
\hline & & $\begin{array}{r}\text { Test de } \\
\text { (calid } \\
\text { varia }\end{array}$ & $\begin{array}{l}\text {-evene } \\
\text { d de } \\
\text { zas) }\end{array}$ & & & ueba t-stc & dent de & gualdad de & medias & \\
\hline & & $F$ & Sig. & $\mathrm{T}$ & g.l. & Sig. & D. de & D. de error & $\begin{array}{r}95 \%(r \\
\text { confi } \\
\end{array}$ & $\begin{array}{l}\text { ivel de } \\
\text { anza) }\end{array}$ \\
\hline & & & & & & & & & Inf. & Sup. \\
\hline $\begin{array}{ll}\text { Evaluación } & \text { de } \\
\text { salida } & \text { de }\end{array}$ & $\begin{array}{c}\text { Varianzas } \\
\text { iguales }\end{array}$ & 1,040 & ,312 & 4,564 & 58 & ,000 & 356,667 & ,78152 & 200,229 & 513,104 \\
\hline $\begin{array}{l}\text { ambos grupos: } \\
\text { experimental y } \\
\text { de control }\end{array}$ & $\begin{array}{l}\text { Varianzas } \\
\text { diferentes }\end{array}$ & & & 4,564 & 58 & ,000 & 356,667 & ,78152 & 200,229 & 513,104 \\
\hline
\end{tabular}

Fuente: Elaboración propia.

En la tabla 2 se observa el resultado de los algoritmos estadísticos en dos muestras independientes. El análisis de los datos se efectúa observando si se cumple tres condiciones: 1) la calidad de las varianzas; 2) diferencia de medias y significatividad bilateral; y 3 ) el valor $t$-student con el nivel de confianza y error estándar. De este modo, se obtuvo según la calidad de varianzas que muestra el test de Levene, la existencia de la condición de igualdad de varianzas, ya que con un nivel de significación de 0,312 y un valor $F$ de 1,040 se evidencia que no existen diferencias significativas entre las varianzas estudiadas; todo lo cual determina la validez de la primera condición. Asimismo, de acuerdo a la prueba t-student de muestras independientes aplicada variables discretas de tipo cuantitativas analizadas, se halló que la diferencia de medias en ambos grupos fue de 3,566 con una significación bilateral de Sig. $0,000<0,05$, con lo cual se cumplió la segunda condición del análisis. Por otro lado, el valor $t$ student con una $\mathrm{T}$ de 4,564 con 58 grados de libertad, considerando que existe una diferencia de error estándar de 0,781 y un $95 \%$ de nivel de confianza se sostiene la representatividad y fiabilidad del análisis efectuado, cumpliendo así la tercera condición. Finalmente, los resultados de los análisis realizados permiten aceptar como verdadera la hipótesis principal de investigación y rechazar la hipótesis nula. Por tanto, se sostiene la existencia de influencia causal y significativa de la implementación de la estrategia del arborigrama en la mejora de la comprensión lectora de los estudiantes.

Igualmente, dentro del sistema de hipótesis se plantearon tres hipótesis derivadas; referidas a cada una de las dimensiones de la variable comprensión de lectura; es decir, la comprensión literal, comprensión inferencial y comprensión crítica o criterial. La primera hipótesis derivada de investigación enuncia que la aplicación de la estrategia del arborigrama influye de forma significativa en la mejora de la comprensión de lectura de textos narrativos, en su dimensión literal en los estudiantes del primer grado del nivel Secundaria de las Instituciones Educativas Públicas en el distrito de La Molina. Esta hipótesis permite generar la hipótesis nula específica a falsar: La aplicación de la estrategia del arborigrama no influye de forma significativa en la comprensión lectora, en su dimensión literal en los estudiantes del primer grado del nivel Secundaria de las Instituciones Educativas Públicas en el distrito de La Molina.

En la tabla 3 se observa el resultado de los algoritmos estadísticos en dos muestras independientes. El análisis de los datos se efectúa observando si se cumple tres condiciones: 1) la calidad de las varianzas; 2) diferencia de medias y significatividad bilateral; y 3) el valor t-student con el nivel de confianza y error estándar. De este modo, se obtuvo según la calidad de varianzas que muestra el test de Levene, la existencia de la condición de igualdad de varianzas, ya que con un nivel de significación de 0,190 y un valor $F$ de 1,757 se evidencia que no existen diferencias significativas entre las varianzas estudiadas; todo lo cual determina la validez de la primera condición. Asimismo, de acuerdo a la prueba $t$-student de muestras independientes aplicada variables discretas de tipo cuantitativas analizadas, se halló que la diferencia de medias en ambos grupos fue de 
Tabla 3

Prueba de hipótesis derivada de la comprensión literal

\begin{tabular}{|c|c|c|c|c|c|c|c|c|c|c|}
\hline \multirow{5}{*}{$\begin{array}{l}\text { Prueba de } \\
\text { salida ítems de } \\
\text { comprensión }\end{array}$} & \multirow[b]{4}{*}{$\begin{array}{c}\text { Varianzas } \\
\text { iguales }\end{array}$} & \multicolumn{2}{|c|}{$\begin{array}{c}\text { Test de Levene } \\
\text { (calidad de } \\
\text { varianzas) } \\
\end{array}$} & \multicolumn{7}{|c|}{ Prueba t-student de igualdad de medias } \\
\hline & & \multirow[t]{2}{*}{$\mathrm{F}$} & \multirow[t]{2}{*}{ Sig. } & \multirow[t]{2}{*}{$\mathrm{T}$} & \multirow[t]{2}{*}{ g.l. } & \multirow{2}{*}{$\begin{array}{c}\text { Sig. } \\
\text { (bilateral) }\end{array}$} & \multirow{2}{*}{$\begin{array}{c}\text { D. de } \\
\text { medias }\end{array}$} & \multirow{2}{*}{$\begin{array}{l}\text { D. de error } \\
\text { estándar }\end{array}$} & \multicolumn{2}{|c|}{$\begin{array}{c}95 \% \text { (nivel de } \\
\text { confianza) }\end{array}$} \\
\hline & & & & & & & & & Inf. & Sup. \\
\hline & & 1,757 & ,190 & 7,521 & 58 &, 000 & 393,333 &, 52296 & 288,652 & 498,015 \\
\hline & $\begin{array}{l}\text { Varianzas } \\
\text { diferentes }\end{array}$ & & & 7,521 & 58 & ,000 & 393,333 & ,52296 & 288,652 & 498,015 \\
\hline
\end{tabular}

Fuente: Elaboración propia.

3,933 con una significación bilateral de Sig. 0,000< 0,05 , con lo cual se cumplió la segunda condición del análisis. Por otro lado, el valor $t$-student con una T de 7,521 con 58 grados de libertad, considerando que existe una diferencia de error estándar de 0,522 y un $95 \%$ de nivel de confianza se sostiene la representatividad y fiabilidad del análisis efectuado, cumpliendo así la tercera condición. Finalmente, los resultados de los análisis realizados permiten aceptar como verdadera la primera hipótesis derivada de investigación sobre la comprensión literal y rechazar la hipótesis nula. Por tanto, se sostiene la existencia de influencia causal y significativa de la implementación de la estrategia del arborigrama en la mejora de la comprensión lectora en el nivel literal de los estudiantes.
La segunda hipótesis derivada de investigación enuncia que la ejecución de la estrategia del arborigrama influye de forma significativa en la comprensión de lectura, en su dimensión inferencial en los estudiantes del primer grado del nivel Secundaria de las Instituciones Educativas Públicas en el distrito de La Molina. Esta hipótesis permite generar la hipótesis nula específica a falsar: La aplicación de la estrategia del arborigrama no influye de forma significativa en la comprensión lectora, en su dimensión inferencial en los estudiantes del primer grado del nivel Secundaria de las Instituciones Educativas Públicas en el distrito de La Molina.

Tabla 4

Prueba de hipótesis de la comprensión inferencial

\begin{tabular}{|c|c|c|c|c|c|c|c|c|c|c|}
\hline & & \multicolumn{2}{|c|}{$\begin{array}{c}\text { Test de Levene } \\
\text { (calidad de } \\
\text { varianzas) } \\
\end{array}$} & \multicolumn{7}{|c|}{ Prueba t-student de igualdad de medias } \\
\hline & & \multirow[t]{2}{*}{$\mathrm{F}$} & \multirow[t]{2}{*}{ Sig. } & \multirow[t]{2}{*}{$\mathrm{T}$} & \multirow[t]{2}{*}{ GI } & \multirow{2}{*}{$\begin{array}{c}\text { Sig. } \\
\text { (bilateral) }\end{array}$} & \multirow{2}{*}{$\begin{array}{l}\text { Dif. de } \\
\text { medias }\end{array}$} & \multirow{2}{*}{$\begin{array}{c}\text { Dif. de error } \\
\text { estándar }\end{array}$} & \multicolumn{2}{|c|}{$\begin{array}{c}95 \% \text { (nivel de } \\
\text { confianza) }\end{array}$} \\
\hline & & & & & & & & & Inf. & Sup. \\
\hline \multirow{2}{*}{$\begin{array}{l}\text { Prueba de } \\
\text { salida ítems de } \\
\text { comprensión } \\
\text { inferencial }\end{array}$} & $\begin{array}{l}\text { Varianzas } \\
\text { iguales }\end{array}$ & 1,692 & ,199 & 5,584 & 58 & ,000 & 333,333 &, 59699 & 213,833 & 452,834 \\
\hline & $\begin{array}{l}\text { Varianzas } \\
\text { diferentes }\end{array}$ & & & 5,584 & 58 & ,000 & 333,333 &, 59699 & 213,833 & 452,834 \\
\hline
\end{tabular}

Fuente: Elaboración propia.

En la tabla 4 se observa el resultado de los algoritmos estadísticos en dos muestras independientes. El análisis de los datos se efectúa observando si se cumple tres condiciones: 1) la calidad de las varianzas; 2) diferencia de medias y significatividad bilateral; y 3 ) el valor t-student con el nivel de confianza y error estándar. De este modo, se obtuvo según la calidad de varianzas que muestra el test de Levene, la existencia de la condición de igualdad de varianzas, ya que con un nivel de significación de 0,199 y un valor $F$ de 1,692 se evidencia que no existen diferencias significativas entre las varianzas estudiadas; todo lo cual determina la validez de la primera condición. Asimismo, de acuerdo a la prueba t-student de muestras independientes aplicada variables discretas de tipo cuantitativas analizadas, se halló que la diferencia de medias en ambos grupos fue de 3,333 con una significación bilateral de Sig. 0, $000<0,05$, con lo cual se cumplió la segunda condición del análisis. Por otro lado, el valor $t$ student con una $\mathrm{T}$ de 5,584 con 58 grados de libertad, considerando que existe una diferencia de error estándar de 0,596 y un 95\% de nivel de confianza se sostiene la representatividad y fiabilidad del análisis efectuado, cumpliendo así la tercera condición. Finalmente, los resultados de los análisis realizados permiten aceptar como verdadera la primera hipótesis derivada de investigación sobre la comprensión inferencial y rechazar la hipótesis nula. Por tanto, se sostiene la existencia de influencia causal y significativa de la 
implementación de la estrategia del arborigrama en la mejora de la comprensión lectora en el nivel inferencial de los estudiantes.

La tercera hipótesis derivada de investigación enuncia que la ejecución de la estrategia del arborigrama influye de forma significativa en la comprensión de lectura, en su dimensión criterial en los estudiantes del primer grado del nivel
Secundaria de las Instituciones Educativas Públicas en el distrito de La Molina. Esta hipótesis permite generar la hipótesis nula específica a falsar: La aplicación de la estrategia del arborigrama no influye de forma significativa en la comprensión lectora, en su dimensión criterial en los estudiantes del primer grado del nivel Secundaria de las Instituciones Educativas de Públicas en el distrito de La Molina.

Tabla 5

Prueba de hipótesis derivada de la comprensión criterial

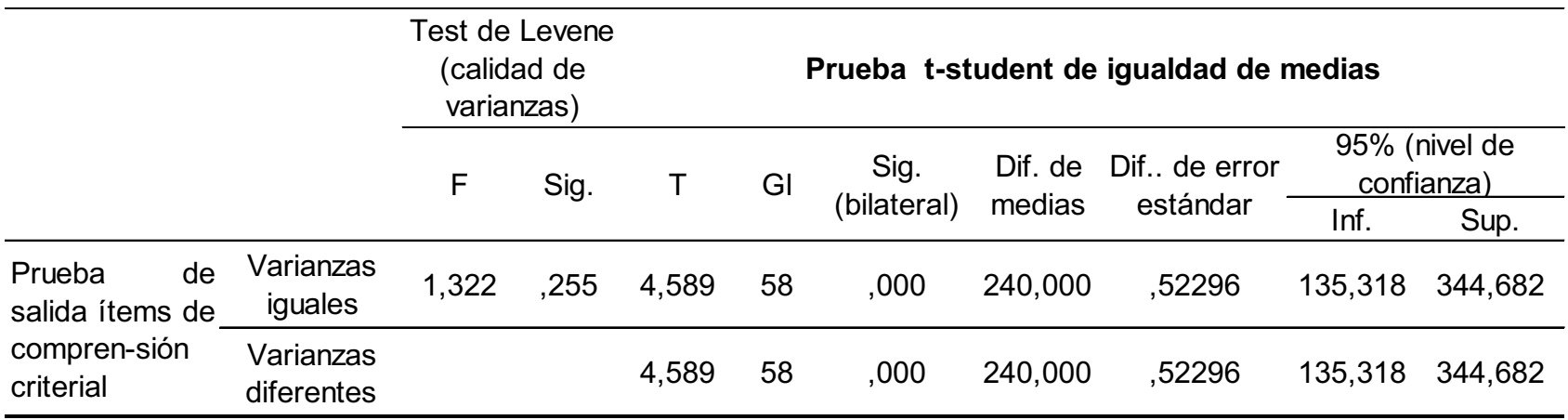

Fuente: Elaboración propia.

En la tabla 5 se observa el resultado de los algoritmos estadísticos en dos muestras independientes. El análisis de los datos se efectúa observando si se cumple tres condiciones: 1) la calidad de las varianzas; 2) diferencia de medias y significatividad bilateral; y 3) el valor $t$-student con el nivel de confianza y error estándar. De este modo, se obtuvo según la calidad de varianzas que muestra el test de Levene, la existencia de la condición de igualdad de varianzas, ya que con un nivel de significación de 0,255 y un valor $F$ de 1,322 se evidencia que no existen diferencias significativas entre las varianzas estudiadas; todo lo cual determina la validez de la primera condición. Asimismo, de acuerdo a la prueba t-student de muestras independientes aplicada variables discretas de tipo cuantitativas analizadas, se halló que la diferencia de medias en ambos grupos fue de 2,400 con una significación bilateral de Sig. 0 , $000<0,05$, con lo cual se cumplió la segunda condición del análisis. Por otro lado, el valor $t$ student con una $\mathrm{T}$ de 4,589 con 58 grados de libertad, considerando que existe una diferencia de error estándar de 0,522 y un $95 \%$ de nivel de confianza se sostiene la representatividad y fiabilidad del análisis efectuado, cumpliendo así la tercera condición. Finalmente, los resultados de los análisis realizados permiten aceptar como verdadera la primera hipótesis derivada de investigación sobre la comprensión criterial y rechazar la hipótesis nula. Por tanto, se sostiene la existencia de influencia causal y significativa de la implementación de la estrategia del arborigrama en la mejora de la comprensión lectora en el nivel criterial de los estudiantes.

En este trabajo de investigación se ha podido demostrar que existe asociación entre las variables de estudio. En tal sentido, como resultado de la indagación, la estrategia del arborigrama permite desarrollar la comprensión lectora en estudiantes del primer grado del nivel Secundaria en la población estudiada. Sin embargo, el estudio presenta algunas limitaciones. La primera limitación es que sólo se aplica al primer grado del nivel Secundaria por lo que las comprobaciones que permiten los resultados obtenidos sólo pueden generalizarse a dicho grupo de estudio en la educación básica de zonas urbanas. Esta situación evidencia que se desconozca la eficiencia de la estrategia del arborigrama en otros grupos preadolescentes y en otros niveles educativos. Por ello, se hace necesario realizar nuevos estudios que puedan analizar los efectos en otros grupos etarios y que adopten un enfoque longitudinal (Salazar-Rodríguez, 2020).

Una segunda limitación está implícita en la metodología de la investigación, por tratarse de un tema educativo. En principio, el diseño de la investigación experimental, del tipo cuasiexperi-mental en el que no existe posibilidad aleatoria de elección de la población objetivo, pero sí de una población accesible ya agrupada por aulas. Este estudio tiene cuestiones implícitas de validez y fiabilidad según la representatividad de la selección muestral que, por lo tanto, cumple con los criterios de operatividad algorítmica del modelo de ecuaciones y estadística descriptiva utilizados.

Esto conlleva a que la tercera limitación sea precisamente la referida a la muestra, pues si se ampliara daría resultados de mayor contundencia, al propio tiempo que se ampliaría el poder de las pruebas estadísticas. Si al delimitar el tema y al definir el tipo de investigación se consigue estar 
dentro de la rigurosidad científica, tampoco esta situación está exenta de sesgo aunque se haya aplicado estudio considerando el total de sujetos del universo.

Muy a pesar de estas restricciones y limitaciones, esta investigación establece primero la identificación de una estrategia didáctica que facilite los procesos de comprensión de lectura. Segundo, determinar la validación de su eficiencia en el desarrollo de habilidades de comprensión de lectura. De ello, se desprende que las técnicas didácticas sustentadas en el enfoque textual iconoglósico, una de las cuales se emplea en la realización del presente estudio son las más significativas. Asimismo, sin ser el objetivo del estudio se observó que la motivación de los estudiantes del grupo experimental respecto a la tarea lectora fueron los que demostraron mayor satisfacción, la cual fue evidenciada en los gestos y actitudes mostrados (Gonzales, 2017).

Por otro lado, el escaso abordaje de investigaciones similares relacionadas con la temática de la presente investigación representa la oportunidad de incorporar propuestas didácticas innovadoras, que se sometan al rigor de la ciencia y técnica respectivamente (Manoli \& Papadopoulou, 2012; Lucas y Miraval, 2017). El estudio ha permitido observar el efecto sobre la comprensión de lectura que una técnica esquemática denominada el arborigrama como estrategia de lectura permite, pues se convierte en un tipo de infografía para el análisis de textos narrativos aplicable a estudiantes pre-adolescente del nivel de secundaria. La estrategia del arborigrama ha mostrado de acuerdo al diseño de aplicación planteado ser una alternativa de intervención pedagógica válida en la educación básica.

\section{Conclusión}

Las conclusiones de la investigación obtenidas a la luz de los resultados y de la discusión del estudio se distribuyen en conclusiones principales y específicas:

La conclusión principal derivada del estudio es que la aplicación de la estrategia del arborigrama a través del módulo didáctico propuesto influye significativamente en el desarrollo de la comprensión lectora en los estudiantes del primer grado del nivel Secundaria de las Instituciones Educativas Publicas en el distrito de La Molina. Los resultados de investigación demuestran el desarrollo de la comprensión lectora en los tres niveles de lectura: el literal, el inferencial y el criterial.

Una segunda conclusión es que la estrategia del arborigrama evidencia que la representación del árbol y sus partes sirven para organizar la información lógicamente en función del análisis de un texto narrativo. Así como combinar imagen, texto, análisis y dibujo artístico personalizado del árbol, lo cual permite motivar al estudiante hacia la realización de la tarea. Asimismo, se ha evidenciado que permite el desarrollo de la criticidad de acuerdo a los resultados del post test en el desarrollo de las preguntas criteriales. La tercera conclusión es que la utilización del arborigrama como estrategia de lectura es eficaz porque desarrolla la toma de conciencia del estudiante-lector sobre los modelos de organización subyacentes en los textos, los cuales son conocidos también como superestructuras y macroestructuras discursivas, las que a su vez facilita en los estudiantes la comprensión lectora global. Una cuarta conclusión es que la estrategia didáctica del arborigrama ejercita y refuerza en el estudiante diversas estrategias cognitivas que favorecen la comprensión lectora como la estrategia de inferencia, la de confirmación, la de cuestionamiento, la de relectura y la de resumen. Otra conclusión es que el módulo didáctico de la estrategia del arborigrama mostró eficacia y efectividad en la promoción de la lectura y posibilitó la comprensión lectora, por lo que se convierte también en un instrumento para el aprendizaje rápido o fast learning y como posibilidad para aplicaciones del enfoque denominado micro learning o microaprendizaje.

Finalmente, se sugiere aplicar la estrategia a través del módulo didáctico a estudiantes del primer y segundo grado para ver influencias y correlaciones en otros grupos de pre adolescentes en instituciones educativas de distintas zonas: zonas rurales, urbanas y urbano-marginales. El módulo de la estrategia didáctica del arborigrama utilizado en este estudio se puede obtener en https://osf.io/56hbs/.

\section{Fuente de financiamiento}

La presente investigación fue autofinanciada.

\section{Contribución de los autores}

El autor participó en todo el proceso de la investigación.

\section{Conflicto de Interés}

Se declara no tener conflicto de interés.

\section{Referencias bibliográficas}

Bean, T. W., Singer, H., Sorter, J., \& Frazee, C. (1986). The Effect of Metacognitive Instruction in Outlining and Graphic Organizer Construction on Students' Comprehension in a Tenth-Grade World History Class. Journal of Reading Behavior, 18(2), 153-169. https://doi.org/10.1080/10862968609547562

Block, E. (1986). The comprehension of second language readers. Tesol Quarterly, 20 (3). https://doi.org/10.2307/3586295

Cain, K. \& Oakhill, J. (2014). Reading comprehen- 
sion and vocabulary: Is vocabulary more important for some aspects of comprehension? L'Année psychologique, 114(4), 647-662. https://doi.org/10.4074/S0003503314004035

Coll, C. (2001). Constructivismo y educación: la concepción constructivista de la enseñanza y el aprendizaje. En C. Coll J. Palacios y A. Marchesi (Comps.), Desarrollo psicológico y educación 2. Psicología de la educación escolar (pp.157177). Madrid: Alianza.

Colliot, T. \& Jamet, E. (2018). Does self-generating a graphic organizer while reading improve students' learning? Computers \& Education, 126, 13-22.

https://doi.org/10.1016/j.compedu.2018.06.028

Derry, S., \& Murphy, D. (1986). Designing systems that train learning ability: from theory to practice. Review of Educational Research, 56, 1-39. Recuperado de:

https://journals.sagepub.com/toc/rera/56/1

Dubois, M. E. (1984). Algunos interrogantes sobre comprensión de la lectura. Lectura y vida, 5(4), 14-19. Recuperado de:

http://www.lecturayvida.fahce.unlp.edu.ar/num eros/a5n4

Düsrteler, J. C. (2002). Visualización de la información: Una visita guiada. Barcelona, Gestión 2000.

Duke, N., \& Pearson, P. (2002). Effective practices for developing reading comprehension. In $A$. Farstrup y S. Samuels (Eds.), What research has to say about reading instruction (pp. 205242). Newark: International Reading Association.

Ehrlich, M. (1999). Metacognitive monitoring of text cohesion in children. In H. Oostendorp y S. Goldman (Eds.). The construction of mental representations during reading (pp.281-301). Mahualh: Lawrence Erlbaum associates.

García Madruga, J.A. (2006). Lectura y conocimiento. Barcelona: Paidós.

Goodman, K. (1996). La lectura, la escritura y los textos escritos: una perspectiva transaccional sociopsicolingüística. Buenos Aires: Lectura y vida, pp. 54-58.

González, G. (2017). Los efectos de los organizadores gráficos en la comprensión lectora de los estudiantes de inglés como lengua extranjera. (Tesis de maestría inédita). Universidad Icesi, Cali, Colombia. Recuperado de:

http://repository.icesi.edu.co/biblioteca_digital/b itstream/10906/83478/1/T01285.pdf

Guerra R. (2017). El libro de los organizadores gráficos. Ecuador: Universidad técnica del Norte. Recuperado de:

https://issuu.com/utnuniversity/docs/ebook_org anizadores_graficos

Izquierdo, A. \& Rodriguez, N. (2020) Using Graphic Organizers to Improve Reading Comprehension and Motivation towards Reading in High School Students (Chapter 4). Mindful Learning: Research on Learner Awareness, Metacognition, and Learning Strategies. Publication of the Institución Universitaria Colombo Americana - ÚNICA, pp.74-99.

Kim, A.-H., Vaughn, S., Wanzek, J., \& Wei, S. (2004). Graphic organizers and their effects on the reading comprehension of students with LD: a synthesis of research. Journal of Learning Disabilities, 37, 105-118. Recuperado de: https://journals.sagepub.com/toc/ldxa/37/2

Levin, J. R., \& Pressley, M. (1983). Understanding mnemonic imagery effects: A dozen "obvious" outcomes. En M. L. Fleming y D. W. Hutton (Eds.), Mental Imagery and learning (pp. 33-51). Englewood Cliffs, New Jersey: Educational Technology Publications.

Loayza, E. (2020). La técnica del arbograma en el desarrollo de habilidades de comprensión de lectura de los estudiantes del primer grado de educación secundaria del distrito de La Molina. (Tesis de maestría inédita). Universidad Nacional de Educación, Lima, Perú. Recuperado de: http://repositorio.une.edu.pe/handle/UNE/4646

Lucas, A., y Miraval, C. (2017). Efecto de los talleres de lectura en el desarrollo de las capacidades de lectura y redacción en alumnos del I ciclo de educación básica - sección Obas. Investigación Valdizana, 8(1), 41-44. Recuperado de: http://revistas.unheval.edu.pe/index.php/riv/arti cle/view/283

Manoli, P. \& Papadopoulou, M. (2012). Graphic Organizers as a Reading Strategy: Research Findings and Issues. Creative Education, 3, 348-356. https://doi.org/10.4236/ce.2012.33055

Mc Daniel, M. A., \& Einstein, G. O. (1991). Reading a series of similar texts: Testing a schema-based learning theory. Bulletin of the Psychonomic Society, 29(4), 297-300. Recuperado de: https://link.springer.com/article/10.3758/BF033 33924

Meyer, B. J. (1984). Text dimensions and cognitive processing. En H. Mandl, N. L Stein y T. Trabasso. Learning and comprehension of text. Hillsdale. New Jersey, Erlbaum.

Novak, J. D., \& Cañas, A. J. (2008). The theory underlying concept maps and how to construct and use them. Technical Report IHMC CmapTools 2006-01 Rev 01-2008, Florida Institute for Human and Machine Cognition. Recuperado de:

http://cmap.ihmc.us/Publications/ResearchPap ers/TheoryUnderlyingConceptMaps.pdf

Paris, S. G., Cross, D. R., \& Lipson, M. Y. (1984). Informed strategies for learning: A program to improve children's reading awareness and comprehension. Journal of Educational psychology, 76(6), 1239. https://doi.org/10.1037/0022-0663.76.6.1239

Paris, S.G., Wasik, B.A. \& Turner, J.C. (1991). The development of strategic readers. En R. Barr, M.L. Kamil, P. Mosenthal \& P.D. Pearson (Eds.), Handbook of reading research (pp. 609-640). White Plains, NY: Longman.

Pearson, P., \& Fielding, L. (1991). Comprehension instruction. In R. Barr, M. Kamil, P. Mosenthal, y 
P. Pearson (Eds.), Handbook of reading research (Vol. 2, pp. 815-860). New York: Longman.

Pérez, S. (2015). Estrategias cognitivas y metacognitivas para la comprensión lectora [Tesis de Magister, Universidad Distrital Francisco José de Caldas]. Repositorio de la Universidad Distrital Francisco José de Caldas, Bogotá. Recuperado de:

https://repository.udistrital.edu.co/handle/1134 9/3951

Prado-Huarcaya, D. y Escalante-López, M. (2020). Estrategias de aprendizaje y la comprensión de textos escritos del idioma inglés. Investigación Valdizana, 14(3), 140-147.

https://doi.org/10.33554/riv.14.3.730

Pressley, M. (1999). Cómo enseñar a leer. Barcelona: Paidós.

Salazar-Rodríguez, A. (2020). Text comprehension at school: Is the application of graphic organizers still important to develop it? EDUSER, 7(2), 110-118. https://doi.org/10.18050/eduser.v7i2.2543

Sarig, G. (1987). High-level reading in the first and in the foreign language: Some comparative process data. Research in reading in English as a second language, 105-120.

Scardamalia, M. \& Bereiter, C. (1984). Development of strategie in texts processing. En H. Mandl, N. L Stein y T. Trabasso. Learning and comprehension of text. Hillsdale. New Jersey, Erlbaum.

Solé, I y Teberosky, A. (2001). La enseñanza y el aprendizaje de la alfabetización: una perspectiva psicológica. Palacios, J., Marchessy, A., Coll, C. (Comp.), Desarrollo psicológico y educación (pp. 461-485). Madrid: Alianza.
Short, E.J. \& Ryan, E.B. (1984). Metacognitive differences between skilled and less skilled readers: Remedianting deficits through story grammar and atribution training. Journal of Educational Psychology, 76, 225-235. https://doi.org/10.1037/0022-0663.76.2.225

Stull, A. T., \& Mayer, R. E. (2007). Learning by doing versus learning by viewing: Three experimental comparisons of learner-generated versus author-provided graphic organizers. Journal of Educational Psychology, 99(4), 808-820. https://doi.org/10.1037/0022-0663.99.4.808

Thayne, S. (2018). Graphic Organizers. The TESOL Encyclopedia of English Language Teaching, 1-7. https://doi.org/10.1002/9781118784235.eelt04 94

Thorndyke, P. W., \& Hayes-Roth, B. (1979). The use of schemata in the acquisition and transfer of knowledge. Cognitive Psychology, 11, 82-106. https://doi.org/10.1016/0010-0285(79)90005-7

Van Dijk, T. \& Kintsch, W. (1983). Strategies of Discourse Comprehension. New York, Estados Unidos: Academic Press.

Villalobos, J. (2020). El uso de las estrategias cognoscitivas en el desarrollo de la comprensión de la lectura de textos escritos en una lengua extranjera. Revista de Lenguas Modernas, (33), 53-82. https://doi.org/10.15517/rlm.v0i33.41080 


\title{
La formación continua de los docentes de educación básica infantil en américa latina: una revisión sistemática
}

\section{The continuous training of early childhood teachers in Latin America: a systematic review}

\author{
Vilma I. Aguirre-Canales ${ }^{1, a, *}$, Julia A. Gamarra-Vásquez ${ }^{1, b, \#}$, Nelly A.M. Lira-Seguín ${ }^{2, c, \%}$, Wilfredo Carcausto ${ }^{1, \mathrm{~d}, \AA}$
}

\begin{abstract}
Resumen
El propósito principal del presente artículo fue llevar a cabo una revisión sistemática de estudios empíricos sobre la formación continua de docentes en los niveles de educación básica infantil en América Latina, dicha información fue recogida de revistas indexadas en relación al tema en la base de datos de Scielo, Scopus, Ebsco y Dialnet, entre los años 2010 al 2019. De acuerdo al análisis de las evidencias recopiladas, en los diferentes estudios se identificaron aspectos como: a) la formación continua como medio de transformación de la práctica pedagógica, b) el desarrollo profesional docente y la cultura investigativa como parte de la formación continua, c) la importancia del desarrollo de los programas de formación continua para potenciar las capacidades pedagógicas de los docentes. Concluyendo que las publicaciones en relación a la formación continua presentan un carácter ascendente, siendo Brasil el país con el mayor número de publicaciones. Así también, se develó que la metodología más utilizada en estos estudios es de enfoque cualitativo y finalmente se demostró que las aplicaciones de los programas de formación continua tuvieron efectos positivos en la modificación de su trabajo diario como docente y la mejora como profesional.
\end{abstract}

Palabras clave: formación continua, transformación de la práctica docente, competencias pedagógicas, sistema educativo.

\begin{abstract}
The main purpose of this article was to carry out a systematic review of empirical studies on the continuous training of teachers at the levels of basic early childhood education in Latin America, this information was collected from journals indexed in relation to the subject in the database of Scielo, Scopus, Ebsco and Dialnet, between the years 2010 to 2019 . According to the analysis of the collected evidence, aspects such as: a) continuous training as a means of transforming pedagogical practice, $b$ ) teacher professional development and research culture as part of continuous training, $c$ ) the importance of developing continuous training programs to enhance the pedagogical capacities of teachers. Concluding that the publications in relation to continuous training present an ascending character, with Brazil being the country with the highest number of publications. Likewise, it was revealed that the methodology most used in these studies is of a qualitative approach and finally it was shown that the applications of continuous training programs had positive effects on the modification of their daily work as a teacher and improvement as a professional.
\end{abstract}

Keywords: continuous training, transformation of teaching practice, pedagogical competences, educational system.

${ }^{1}$ Universidad Cesar Vallejo, Perú

${ }^{2}$ Pontificia Universidad Católica Del Perú, Perú

E-mail, ’vaguirrec@ucvvirtual.edu.pe, bjgamarrava@ucvvirtual.edu.pe, cnlira@pucp.edu.pe, 'wcarcaustocalla@ucvvirtual.edu.pe

Orcid ID: "https://orcid.org/0000-0001-5258-8166, " https://orcid.org/0000-0002-4725-2913, \%https://orcid.org/0000-0002-9303-356X, \&https://orcid.org/0000-0002-3218-871X 
Introducción

En los primeros decenios del presente siglo, los docentes asumen retos como la globalización, la revolución digital, donde las costumbres y modo de vida de las personas se han visto modificados por el avance permanente e incontenible de las tecnologías digitales e internet, así mismo enfrentan nuevos escenarios en la práctica cotidiana con innovadoras formas de enseñanza aprendizaje (Maldonado-Ramirez, 2020; Viñals y Cuenca, 2016).

El papel de la formación continua es vigente, el docente analiza los efectos de las innovaciones, adaptando su trabajo y desarrollando nuevas estrategias de enseñanza atendiendo a las necesidades del contexto individual así como teorías de renovación permanente en conocimientos pedagógicos (Núñez-Rojas, 2019; GonzálezFernández, Zabalza-Cerdeiriña, Medina-Domínguez y Medina-Rivilla, 2019) por lo que la formación permanente se ha transformado en un proceso encaminado a la sustitución y actualización de competencias inicialmente alcanzadas, siendo de necesidad actualizarlos para responder a los cambios del contexto social (Chávez y Vieira, 2020; Aneas Novo, Sánchez Rodríguez \& Sánchez Rivas, 2019; Pérez-Van-Leenden, 2019; Escudero, Gonzales y Ramírez, 2018). De esta manera, la formación continua es considerada relevante en las políticas educativas, decisiva para la modernización de los organismos escolares logrando alcanzar la eficacia educativa (Guadalupe, León, Rodríguez y Vargas, 2017).

Cabe resaltar que la formación continua comprende dimensiones como la Educativa se encuentra relacionada a los procesos de actualización docente a partir de los cambios curriculares y los procesos de formación continua de acuerdo a la realidad educativa. También, se considera la dimensión pedagógica, como la aplicabilidad de los conocimientos aprendidos en su labor docente, en lo que respecta a la dimensión humana, se considera la relevancia que tuvo el desarrollo de los proyectos de formación continua en el desarrollo personal y laboral del docente y finalmente la investigativa relacionada a la innovación educativa a partir de las habilidades cognitivas alcanzadas en los procesos de la formación continua. (Quiroz, 2015). Además, el presente artículo aborda el ámbito educativo básico infantil clasificado por niveles tal es el caso del nivel inicial o infantil o pre escolar, así también el nivel primaria y secundaria.

La formación continua de los docentes es aquella que viabiliza el desarrollo de competencias pedagógicas propias del ejercicio profesional, se inserta como elemento indispensable para el mejoramiento continuo de la práctica educativa, (Jiménez, González y Tornel, 2018). Así mismo, la formación continua se conceptualiza como un proceso sistemático, consecuente y organizado en el cual los docentes en servicio participan de forma individual o colectiva en procesos de formación críticamente reflexiva propiciando el desarrollo de competencias profesionales.

Son diversas las informaciones recopiladas sobre este tema en artículos de divulgación científica, como originales y revisión, sin embargo, recientemente se ha efectuado una revisión de la literatura sobre formación continua de los profesores de educación básica del nivel primaria en el área de Educación física, cuyo análisis develó la necesidad de ir actualizando de forma permanente los conocimientos pedagógicos sobre la didáctica para la mejora en la enseñanza de esta área en el contexto colombiano. (Gómez, 2018) También, la revisión literaria sobre la formación permanente del docente considerado como un camino para perfeccionar la calidad de la educación infantil, enfocándose de manera directa en el crecimiento propio y profesional del docente que participa en estos programas, quien genera a través del proceso enseñanza impacto positivo sobre la población infantil y la calidad de su educación (Ceballos-Lopez: Susinos- Rada; y García-Lastra, 2018). Así mismo, la revisión sobre la eficacia de los programas de formación de docentes en servicio en la educación básica en Latinoamérica, en la que tuvo como base de datos revistas internacionales indexadas recientes, centrando su análisis en los aportes de la formación docente en la etapa inicial y permanente, para desarrollar competencias en gestión, pedagógicas e investigativas que mejoran la calidad educativa en los países latinoamericanos (Cuadra \& Catalán, 2016). También, se realizó un análisis crítico sobre las políticas y modelos de formación continua peruano, realizado por el MINEDU, quien a través del desarrollo de programas de capacitación actualizaron los conocimientos pedagógicos de los docentes para la transformación de sus prácticas en el aula y la mejora de los aprendizajes Galazzi, Gomez y Vázquez 2018).

Del compendio de artículos referidos al tema de investigación, se determina que, si bien se cuentan con estas revisiones de literatura sobre la formación continua de los docentes en educación básica infantil en América Latina, sin embargo, no se dispone de muchas fuentes de revisión sistemática sobre este tema, sus dimensiones y estrategias como el acompañamiento pedagógico y la capacitación docente que incide directamente en la trasformación de la práctica pedagógica del profesorado. En los últimos cuatro lustros del siglo $\mathrm{XXI}$ este campo de estudio adquiere relevancia ya que constituye no solo un reto si no también un compromiso con la sociedad de mejorar cada vez más la educación hasta alcanzar la calidad que tanto se anhela. Así mismo, es de interés para los docentes, directivos y especialista conocer la realidad de la formación continua en países Latinoamericanos, con la finalidad de ir actualizando sus conocimientos y analizar alternativas 
para el abordaje en esta materia.

El artículo tuvo como propósito revisar de forma sistemática sobre la formación continua de los docentes de educación básica infantil en América Latina. Asimismo, se buscó estimar la cantidad de artículos científicos sobre formación continua de docentes de educación básica infantil según el año de publicación de la base de datos de las revistas Scielo, Scopus, Ebsco y Dialnet, así como también el número de autores de las publicaciones, relación de los títulos con el tema de estudio, el país de origen de las publicaciones teniendo en cuenta que correspondan a América Latina, enfoque y diseño metodológico, muestras y resultados relevantes.

\section{Método}

La revisión sistemática, es un proceso de investigación que utiliza como estrategia la revisión consecuente, objetiva y exhaustiva de estudios empíricos que se han realizado sobre un determinado problema. (Leite, Padilha, Soares y Cecatti, Jose G, 2019; Labarca, Uribe, Majid, Folch, \& Fernandez-Bussy, 2020). Por consiguiente, el método utilizado en el presente estudio fue Prisma para revisiones sistemáticas, el cual inicia con un proceso de revisión preliminar con la búsqueda de información por el título del tema hasta el final identificando estudios de mayor relevancia. Esta metodología de revisión generalmente es utilizada por las ciencias médicas, sin embargo, recientemente se ha identificado estudios en las ciencias sociales como metodología para la realización de revisiones sistemáticas de literatura científica. (Cajal, Gervilla y Montaño, 2020).

\section{Criterios de inclusión y exclusión}

En este sentido, el presente artículo ha tenido en cuenta los siguientes criterios de inclusión en su proceso de búsqueda de revisión: 1) Estudios relacionados a educación y ciencias sociales 2)Año de publicación entre 2010 y 2019, 3) Disponibles en idioma Español, inglés y portugués 4) Tipo de publicación (artículos científicos) y de acceso (abierto y completo), 5) Desarrollados en la temática de la formación continua de profesores de educación infantil, primaria y secundaria 6) Estudios con diseños cuantitativos y cualitativos. Así también los criterios de exclusión fueron: 1) La categorización, es decir estudios que no correspondan a la rama de educación y ciencias sociales 2) Estudios no empíricos, tales como notas, ensayos, conferencias y comentarios, 3) Artículos publicados antes del año 2010 y después del 2019 , 4) Estudios en idiomas diferentes al español, inglés y portugués 5) Artículos repetidos o duplicados 6) Estudios de formación continua en docentes de educación superior y de otras profesiones diferentes a la educación. (Creswell \& Poth, 2018).

\section{Protocolo de búsqueda}

La búsqueda preliminar se desarrolló durante el mes de agosto del 2020, a través de la Web a partir de la década de los noventa, fecha en que se inicia los programas de formación continua, centrando mayor atención en aquellos relacionados a la formación continua de educación básica infantil (Cuadra \& Catalán, 2016), esta búsqueda fue efectuada en la base de datos de las revistas Scielo, Scopus, Ebsco y Dialnet utilizando descriptores o palabras combinadas en los idiomas español, inglés y portugués, cuyos resultados de búsqueda se observan en la tabla 1. A partir de ello, se aplicaron criterios de inclusión y exclusión propuestos en este estudio, mediante diversos filtros identificados en las bases de datos para la obtención de resultados más precisos que permitan la elección de los artículos para ser incluidos en esta revisión.

Tabla 1: Resultado de la búsqueda preliminar en la base de datos de revistas, utilizando palabras combinadas e idioma español, inglés y portugués

\begin{tabular}{|c|c|c|c|c|c|}
\hline \multirow{2}{*}{ Palabras combinadas en español, inglés y portugués } & \multicolumn{4}{|c|}{ Base de datos } & \multirow{2}{*}{ Total } \\
\hline & Scielo & Scopus & Ebsco (*) & Dialnet & \\
\hline Formación continua y educación infantil & 9 & 1 & 616 & 144 & 770 \\
\hline Formación continuada y educación básica & 23 & 0 & 148 & 331 & 502 \\
\hline Formación permanente y educación infantil & 13 & 1 & 782 & 201 & 997 \\
\hline Formación posgraduada y educación básica & 2 & 0 & 9 & 8 & 19 \\
\hline Continuous training and early childhood education & 8 & 24 & 1208 & 18 & 1258 \\
\hline Continuous training and basic education & 44 & 536 & 3146 & 108 & 3834 \\
\hline Permanent training and early childhood education & 3 & 12 & 144 & 20 & 179 \\
\hline Postgraduate training and basic education & 46 & 957 & 2722 & 40 & 3765 \\
\hline Formação contínua de professores e educação infantil & 6 & 0 & 6362 & 57 & 6425 \\
\hline Formação continuada de professores e educação básica & 55 & 0 & 11457 & 207 & 11719 \\
\hline Formação permanente e educação infantil & 6 & 0 & 9931 & 10 & 9947 \\
\hline formação pós-graduação e educação básica & 31 & 0 & 19541 & 130 & 19702 \\
\hline Total & 246 & 1531 & 56066 & 1274 & 59117 \\
\hline
\end{tabular}

$\left.{ }^{*}\right)$ En la base de datos de Ebsco se utilizó el operador booleano AND y comillas para mejorar la búsqueda de información. 
Elegibilidad e inclusión de artículos para revisión y síntesis

Se realizó una lectura preliminar de los artículos seleccionados a partir del título, el resumen, la metodología y los resultados a que arribaron los investigadores, posterior a ello se realizó una lectura exhaustiva y completa de estos estudios, identificando ideas y datos relevantes que faciliten el avance de la presente investigación. Los artículos clasificados para la revisión sistemática fueron organizados en una matriz de Microsoft Excel, teniendo como marco de referencia 1) el año de publicación, 2) el autor o los autores, 3) el título del artículo, 4) el diseño metodológico 5) muestra de estudio 6) el resultado principal.

\section{Resultados}

En la tabla 1 tenemos el recuento de la búsqueda preliminar, en el cual se identificaron 59117 estudios como resultado de la aplicación de las palabras claves tanto en el idioma español, (Formación continua y Educación infantil; Formación continuada y Educación básica; Formación permanente y Educación infantil y Formación posgraduada y Educación básica) en inglés (Continuous training and early childhood education; Continuing training and basic education; Permanent training and Early childhood education and Postgraduate training and Basic education) y en portugués Formação contínua de professores e educação infantil, Formação continuada de professores e educação básica, Formação permanente e educação infantil y formação pós- graduação e educação básica. Estos estudios fueron hallados en la base de datos de las revistas seleccionadas para la presente investigación $($ Scielo, $=246$, Scopus $=1531$, Ebsco $=56066 \mathrm{y}$ Dialnet $=1274$ ) considerando dentro de ello todo tipo de investigaciones y estudios relacionados al tema.

Posteriormente, se aplicaron filtros o criterios de inclusión y exclusión considerados en este estudio, el primer criterio utilizado fue el filtro fue la categorización, en el que sólo fueron considerados aquellos estudios correspondientes a las disciplinas de educación y ciencias sociales $(n=53096)$, del mismo modo se aplicó el filtro correspondiente al año de publicación, en el que se tuvo en cuenta el periodo del 2010 al $2019(n=1099)$. Seguidamente, se utilizó del idioma de estudios en los idiomas español, inglés y portugués $(n=644)$ y de igual manera se aplicó el filtro para el tipo de publicación y acceso en el que sólo fueron considerados artículos completos y acceso abierto $(n=2356)$. Por otra parte, en cuanto al filtro de temática y contexto fueron seleccionados aquellos artículos vinculados con la formación continua y el contexto de educación básica infantil, primaria y secundaria $(n=1788)$. Finalmente, fueron elegidos los artículos basados en metodología de investigación científica $(n=113)$, descartando estudios duplicados $(n=3)$ y según el enfoque y diseño metodológico $(n=115)$ obteniendo como resultado final 16 artículos para el estudio, tal como podemos observar en el diagrama de flujo (Fig. 1)

Fig. 1. Diagrama de Resultados de pesquisa y clasificación de artículos aplicando criterios de inclusión y exclusión

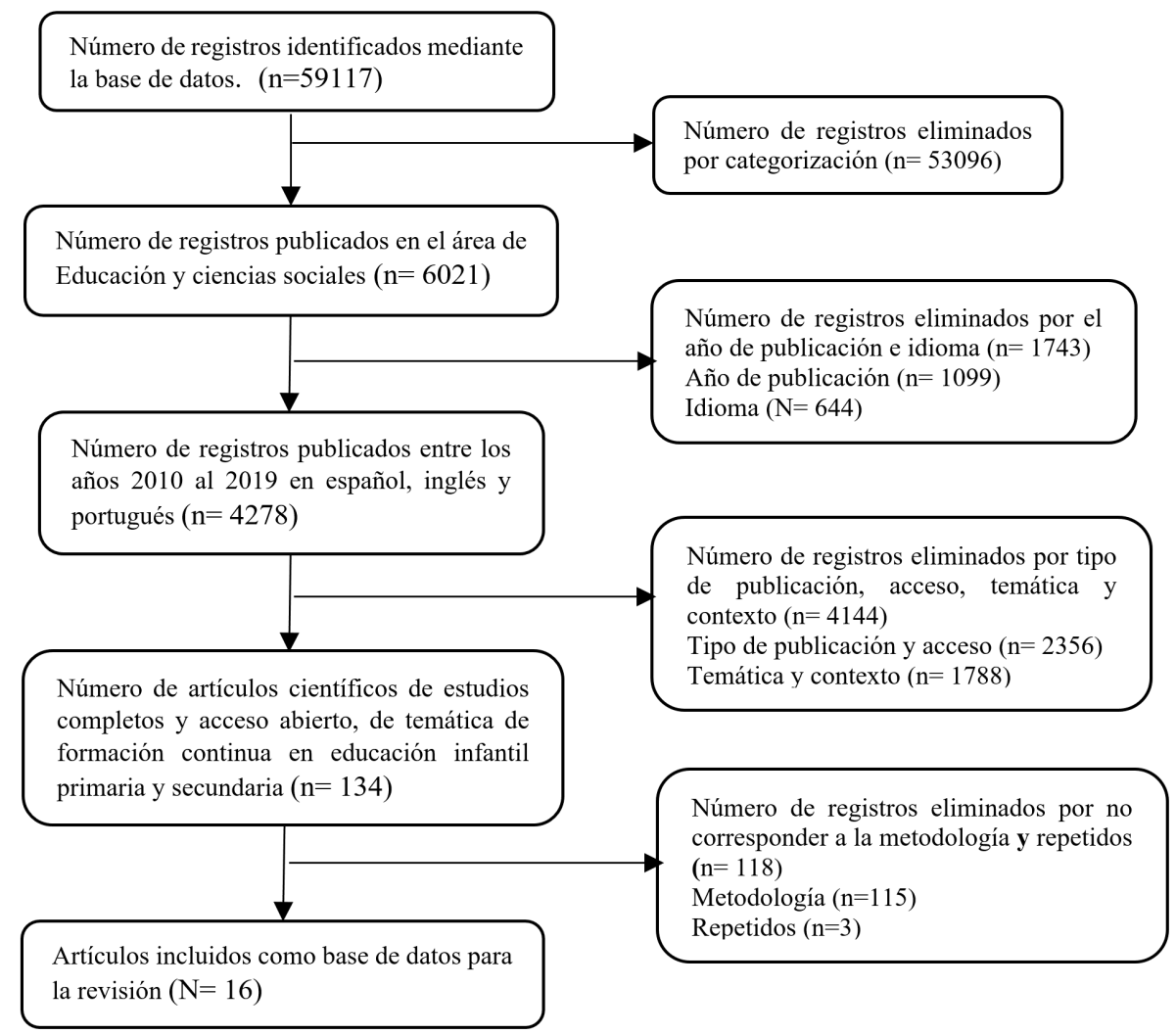


Cantidad de estudios según el año de publicación, autores y países de procedencia. En el periodo de revisión comprendió 10 años (2010 al 2019) fueron seleccionados 16 artículos científicos para la presente revisión, siendo los años 2013, 2014 y 2017 el espacio de tiempo donde la producción no alcanzo a superar las dos publicaciones por año, Aun más se observa esta tendencia de baja en los años 2015 y 2016 en el que se publica un artículo por año considerándose estos años el de mayor declive de producciones científicas, sin embargo, entre los años 2018 y 2019 se muestra una inclinación en aumento siendo estos los de mayor producción científica publicada en las bases de datos seleccionados. (Fig. 2)

Fig. 2. Publicaciones relacionadas a la formación continua de docentes de educación básica infantil en el periodo 2010 al 2019

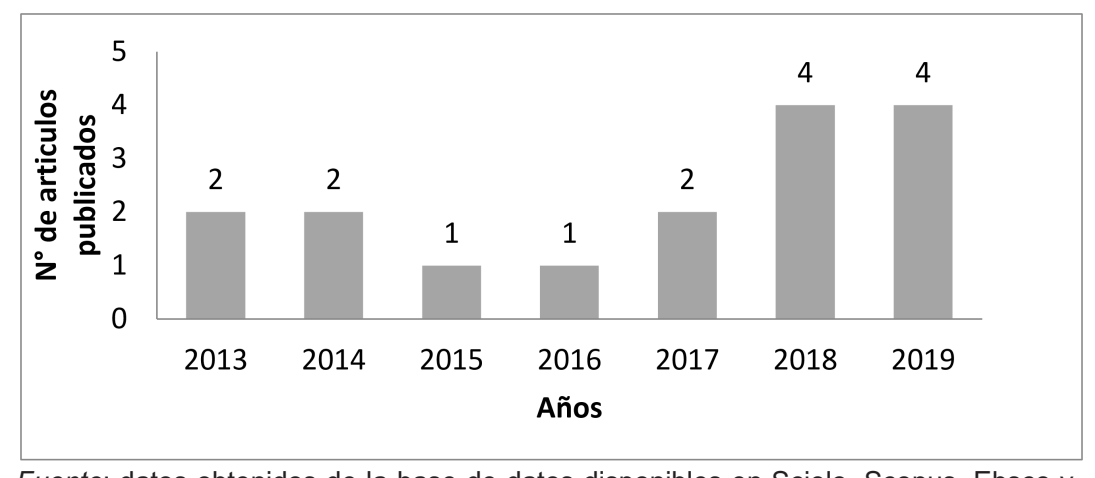

Fuente: datos obtenidos de la base de datos disponibles en Scielo, Scopus, Ebsco y

Dialnet

En lo concerniente al número de autores de las publicaciones, se observa en la tabla 2, los 16 estudios seleccionados para la presente investigación, 7 de ellos tuvieron un solo autor: Castelo (2016); Capistrán (2018); Fernández (2014); Gureski (2019); Oliveira (2014); Quintanilla (2019) y Rocha (2017), así también, 4 estudios fueron identificados con dos autores: Cardoso y De Oliveira (2013); De Azevedo y Pacheco (2018); Duarte y Hermes (2013); Marlow y Do Rosário (2018), sin embargo, 5 artículos fueron registrados con 3 autores: Ballesteros, Mercado y García (2019); Cerchiaro, Vargas y Barras (2019); De Castro, Zanon y Albuquerque (2017); Días, Sousa y Silveira (2018) y Díaz, Luna y Jiménez (2015).

En lo que respecta a la relación de los títulos con el tema de estudio, 6 autores utilizaron la denominación de formación continua en sus artículos al referirse a las acciones realizadas para potenciar las competencias pedagógicas de los educadores para mejorar su práctica educativa, estos son: Cardoso y De Oliveira (2013); Duarte y Hermes (2013); Gureski (2019); Oliveira (2014); Quintanilla (2019) y Rocha (2017), siendo esta la denominación con mayor incidencia por parte de los autores, del mismo modo, 5 autores utilizaron la denominación formación docente al referirse al tema, tales como: Ballesteros, Mercado y García (2019); Capistrán (2018); De Azevedo y Pacheco (2018); Díaz, Luna y Jiménez (2015) y Fernández (2014), de la misma forma, 5 autores utilizaron otras designaciones como: educación continua, Castelo (2016); formación del profesorado, Días, Sousa y Silveira (2018); formación de maestras, Cerchiaro, Vargas y Barras (2019); desarrollo docente, Marlow y Do Rosário (2018), y entrenamiento continuo, De Castro, Zanon y Albuquerque (2017), sin embargo, a pesar de tener otras denomina- ciones en sus títulos, en el contenido abordan la temática de la investigación.

En relación a los niveles educativos utilizados en los títulos, 4 autores utilizaron la denominación educación infantil o primera infancia para referirse a los estudiantes de educación inicial, jardín o kindergarten, estos fueron: Cerchiaro, Vargas y Barras (2019); Días, Sousa y Silveira (2018); Duarte y Hermes (2013) y Gureski (2019), del mismo modo, 3 autores utilizaron la denominación educación básica para referirsea los niveles de primaria y secundaria, dentro de ellos tenemos a: Capistrán (2018); De Castro, Zanon y Albuquerque (2017); Díaz, Luna y Jiménez (2015), no obstante, 9 autores en sus títulos no consideraron la denominación de educación básica o infantil, sin embargo, a pesar de utilizar otras denominaciones, los contenidos de los artículos abordan la temática propuesta en este estudio, ellos fueron: Ballesteros, Mercado y García (2019); Cardoso y De Oliveira (2013); Castelo (2016); De Azevedo y Pacheco (2018); Marlow y Do Rosário (2018); Oliveira (2014); Quintanilla (2019) y Rocha (2017).

El estudio se circunscribió geográficamente en América Latina y los países que lo conforman, sin embargo, los artículos seleccionados para la presente revisión corresponden solo a tres de ellos, siendo Brasil el país que más publicaciones ha tenido en este periodo de tiempo obteniendo como resultado 12 artículos, estos corresponden a: Cardoso y De Oliveira (2013); Castelo (2016); De Azevedo y Pacheco (2018); De Castro, Zanon y Albuquerque (2017); Días, Sousa y Silveira (2018); Duarte y Hermes (2013); Fernández (2014); Gureski (2019); Marlow y Do Rosário (2018); Oliveira (2014); Quintanilla (2019) y Rocha (2017) seguido de México quien publico 3 artículos cuyos 
Tabla 2. Distribución de los autores, año de publicación, títulos de estudio, y país de origen

\begin{tabular}{|c|c|c|c|}
\hline Autor(es) & Año & Titulo & País \\
\hline $\begin{array}{l}\text { Ballesteros Ibarra María Lorena } \\
\text { Mercado Varela Martín Alonso } \\
\text { García Vázquez Nancy Janett }\end{array}$ & 2019 & $\begin{array}{l}\text { La formación docente en línea: Experiencias con MOOCs en } \\
\text { Sonora (México) }\end{array}$ & México \\
\hline Capistrán Gracia Raúl W. & 2018 & $\begin{array}{l}\text { Necesidades de formación docente de los maestros de } \\
\text { Educación Artística a nivel Secundaria del Instituto de } \\
\text { Educación de Aguas Calientes. }\end{array}$ & México \\
\hline $\begin{array}{l}\text { Cardoso Urzetta Fabiana } \\
\text { De Oliveira Cunha Ana Maria }\end{array}$ & 2013 & $\begin{array}{l}\text { Análisis de una propuesta colaborativa de formación continua } \\
\text { de profesores de ciencias en perspectiva de desarrollo } \\
\text { profesional docente. }\end{array}$ & Brasil \\
\hline Castelo Branco Ana Cássia & 2016 & $\begin{array}{l}\text { Educación continua y conocimiento experiencial de los } \\
\text { docentes escuelas municipales en São Luís: implicaciones } \\
\text { teórico-prácticas. }\end{array}$ & Brasil \\
\hline $\begin{array}{l}\text { Cerchiaro Ceballos Elda } \\
\text { Vargas Romero Heilen } \\
\text { Barras-Rodríguez Rafael }\end{array}$ & 2019 & $\begin{array}{l}\text { Resultados de un programa educativo para la formación de } \\
\text { maestras de la primera infancia. }\end{array}$ & Colombia \\
\hline $\begin{array}{l}\text { De Azevedo Martins José Pedro } \\
\text { Pacheco Schnetzler Roseli }\end{array}$ & 2018 & $\begin{array}{l}\text { Formación docente en educación ambiental crítica centrado } \\
\text { en la investigación-acción y la asociación colaborativa }\end{array}$ & Brasil \\
\hline $\begin{array}{l}\text { De Castro Wiziack Suzete } \\
\text { Zanon Angela María } \\
\text { Albuquerque Vargas Icleia } \\
\end{array}$ & 2017 & $\begin{array}{l}\text { Entrenamiento continuo de profesores en educación y } \\
\text { sostenibilidad ambiental como política pública para educación } \\
\text { básica en Brasil. }\end{array}$ & Brasil \\
\hline $\begin{array}{l}\text { Dias Costa Efigênia Maria } \\
\text { Sousa Montenegro Fabrícia } \\
\text { Silveira María de Fátima }\end{array}$ & 2018 & $\begin{array}{l}\text { Formación del profesorado y condiciones laborales en la } \\
\text { educación infantil }\end{array}$ & Brasil \\
\hline $\begin{array}{l}\text { Díaz Barriga, Ángel } \\
\text { Luna Miranda, Ana Bertha } \\
\text { Jiménez Vásquez, Mariela Sonia } \\
\end{array}$ & 2015 & $\begin{array}{l}\text { La Formación Docente para la Reforma Integral de Educación } \\
\text { Básica en el nivel primaria. La pertinencia pedagógica del } \\
\text { diplomado para docentes del primero a sexto grado. }\end{array}$ & México \\
\hline $\begin{array}{l}\text { Duarte Pillotto Silvia Sell } \\
\text { Hermes de Araujo Patricia Kricheldorf }\end{array}$ & 2013 & $\begin{array}{l}\text { El blog como posibilidad de aprendizaje y nuevos retos en el } \\
\text { contexto de formación continua para profesores de educación } \\
\text { infantil. }\end{array}$ & Brasil \\
\hline Fernández Carla Helena & 2014 & $\begin{array}{l}\text { Relaciones entre la investigación y la formación docente } \\
\text { permanente: El conocimiento necesario para la diversidad. }\end{array}$ & Brasil \\
\hline Gureski Rodrigues Daniela & 2019 & $\begin{array}{l}\text { La Formación Continua del profesor de Educación Infantil en } \\
\text { Educación Ambiental. }\end{array}$ & Brasil \\
\hline Oliveira Cardoso Sandra & 2014 & $\begin{array}{l}\text { La formación continua de profesores como respuesta para la } \\
\text { equidad y el éxito educativo: evaluación, Tic y diferenciación: } \\
\text { Un estudio de caso. }\end{array}$ & Brasil \\
\hline Quintanilla Corrêa Carla Patrícia & 2019 & $\begin{array}{l}\text { Formación Continua de profesores de Matemáticas, Física y } \\
\text { Química: la experiencia de un programa Iniciación docente } \\
\text { brasileña. }\end{array}$ & Brasil \\
\hline Rocha Tuma Viana Kemle Senhorinha & 2017 & $\begin{array}{l}\text { Formación Continua del profesorado a través del Programa } \\
\text { Pacto Nacional para Alfabetización en la edad adecuada - } \\
\text { Pnaic y su contribución a la práctica pedagógica docente. }\end{array}$ & Brasil \\
\hline $\begin{array}{l}\text { Santos Marlow Marcia Zschornack } \\
\text { Do Rosário Lima Valderez Marina }\end{array}$ & 2018 & $\begin{array}{l}\text { Contribuciones al desarrollo docente en el contexto de un } \\
\text { programa de formación docente. }\end{array}$ & Brasil \\
\hline
\end{tabular}

\section{Abordaje metodológico y resultado}

En la metodología se ha tenido en cuenta el enfoque, el diseño, la muestra y los resultados de los estudios, como se visualiza en la tabla 3 , el enfoque recurrente corresponde al cualitativo $(n=$ $13)$, los diseños que corresponden a este enfoque fueron: estudios de caso $(n=5)$; investigación acción $(n=3)$ y el descriptivo $(n=1)$, sin embargo, una cantidad considerable $(n=4)$ no precisó el diseño de estudio en sus artículos. También se identificaron estudios con enfoque cuantitativo $(n=$ 1), pero el diseño no precisa en su investigación. EI enfoque mixto igualmente fue utilizado por dos investigadores $(n=2)$ en estos estudios, teniendo como diseño de investigación el exploratorio. (Creswell, 2018).

Las muestras seleccionadas para estos estudios 
son docentes de educación básica infantil, recibiendo la denominación de docentes en trece estudios $(n=13)$, sin embargo, en 3 estudios identificados se refieren a los docentes como: maestros $(n=1)$ profesores $(n=1)$ y alfabetizadores $(n=1)$ refiriéndose a los mismos profesionales en la educación. Por otro lado, las muestras de 2 estudios estuvieron también constituida por: gerentes $(n=1)$ al referirse a los directores de las instituciones educativas, directores $(n=1)$ coordinadores $(n=2)$, tal como se puede observar en la tabla 3.

En la tabla 3 nos muestra los principales resultados de este conglomerado de estudios respecto al tema de investigación, de los cuales siete $(n=7)$ de los artículos concluyen señalando que la formación continua es un proceso mediante la cual se fortalece los conocimientos pedagógicos de los docentes para transformar su práctica durante el desarrollo de la enseñanza de los estudiantes, mejorando de esta manera el aprendizaje de los estudiantes. También, seis $(n=6)$ estudios develaron en sus resultados que la formación continua favorece su desarrollo profesional docente, mediante la actualización y el perfeccionamiento de su práctica pedagógica consigue la ampliación del ejercicio de sus funciones permitiendo desenvolverse como especialistas, consultores o formadores, y por último tres artículos $(n=3)$ manifiestan que el desarrollo de los programas formación continua tuvieron un impacto positivo en las competencias pedagógicas de los docentes, como el desarrollar la cultura investigativa entre los docentes, por lo tanto deben ser aplicados como política educativa de los estados.

Tabla 3: Distribución de las muestras y resultados de los estudios comprendidos en el presente artículo

\section{Enfoque/diseño Muestra Breve descripción del resultado}

\begin{tabular}{|c|c|c|}
\hline $\begin{array}{l}\text { Enfoque: } \\
\text { Cualitativo } \\
\text { Diseño: No } \\
\text { precisa }\end{array}$ & 6 docentes & $\begin{array}{l}\text { Los hallazgos evidencian una actitud abierta de los profesores que los capacitaron } \\
\text { en línea; sin embargo, su voluntad de participar en él ha sido influenciada por sus } \\
\text { experiencias previas en esta modalidad. Asimismo, los hallazgos muestran la } \\
\text { necesidad de promover un compromiso conjunto, entre las autoridades educativas } \\
\text { y los docentes, para implementar estrategias masivas exitosas de formación } \\
\text { permanente. }\end{array}$ \\
\hline $\begin{array}{l}\text { Enfoque: Mixto } \\
\text { Diseño: } \\
\text { Exploratorio }\end{array}$ & $103 r$ & $\begin{array}{l}\text { La mejora de la enseñanza de la educación artística requiere del perfeccionamiento } \\
\text { de los docentes, ya que mediante ello se atiende a las reformas educacionales que } \\
\text { buscan mejorar la enseñanza de los estudiantes. }\end{array}$ \\
\hline $\begin{array}{l}\text { Enfoque: } \\
\text { Cualitativo } \\
\text { Diseño: Estudio }\end{array}$ & 50 docentes & $\begin{array}{l}\text { Los programas de educación continua permiten analizar la práctica docente } \\
\text { mediante la actualización de los conocimientos Así como favorecer un desempeño } \\
\text { profesional más alineado a los nuevos tiempos. }\end{array}$ \\
\hline
\end{tabular}

de casos

Enfoque: $\quad 48$ profesores

Diseño: Estudio

de casos
Se contemplan aspectos relevantes para la concurrencia de la educación continua en la escuela y su trascendencia para la docencia.

8 coordinadores

\section{Enfoque:}

Cualitativo

Diseño:

Investigación

acción

\section{Enfoque:}

Cualitativo

Diseño:

Investigación

acción

\section{Enfoque:}

Cualitativo

Diseño:

Descriptivo

\section{Enfoque: Mixto}

Diseño:

Exploratorio
Los resultados al término del programa muestran cambios relevantes en el trabajo pedagógico de los docentes, quienes promueven estrategias de aprendizaje autónomo y colaborativo con los infantes para alcanzar competencias prevista. Por lo que recomienda que este modelo de programas se debe impartir con otros docentes para mejorar su enseñanza.

Los resultados muestran que, debido a la investigación y socialización de las experiencias docentes, los docentes cambiaron gradualmente sus prácticas docentes, inicialmente puntuales y compartimentadas. En el transcurso del trabajo, estas prácticas fueron cambiadas con la inclusión de temas socio-ambientales abordados de manera crítica, contextualizada, permanente, continua y participativa.

Los resultados develan que la formación permanente en los maestros genera 1180 docentes deseos de transformación de sus escuelas donde laboran para convertirla en "Escuela sostenible" como resultado de los conocimientos adquiridos.
Los resultados muestran que los cursos de educación continua se llevan a cabo descontextualizados de la realidad, mientras que la necesidad de los docentes, muestran solo los aspectos técnicos de la formación. 


\begin{tabular}{|c|c|c|}
\hline $\begin{array}{l}\text { Enfoque: } \\
\text { Cualitativo } \\
\text { Diseño: No } \\
\text { precisa }\end{array}$ & docentes & $\begin{array}{l}\text { La formación continua aplicada a los docentes del nivel primaria, genero mayor } \\
\text { impacto en las docentes de género femenino que en el masculino, concluyendo que } \\
\text { las mujeres tienen mayor adaptabilidad en los procesos formativos. }\end{array}$ \\
\hline $\begin{array}{l}\text { Enfoque: } \\
\text { Cualitativo } \\
\text { Diseño: } \\
\text { Investigación } \\
\text { acción }\end{array}$ & 35 docentes & $\begin{array}{l}\text { El blog puede ser utilizado para la práctica pedagógica con el fin de que los niños, } \\
\text { profesores y lector en general interactúen en el uso de un espacio donde puedan } \\
\text { intercambiar ideas y discutir proyectos, haciendo del entorno virtual un espacio de } \\
\text { conversaciones colectivas. }\end{array}$ \\
\hline $\begin{array}{l}\text { Enfoque: } \\
\text { Cualitativo } \\
\text { Diseño: No } \\
\text { precisa }\end{array}$ & 11 docentes & $\begin{array}{l}\text { La investigación educativa es un camino para la transformación de la práctica } \\
\text { pedagógica, mediante ella se busca dar respuesta a las necesidades formativas, } \\
\text { por ello se debe fomentar la cultura investigativa en los docentes. }\end{array}$ \\
\hline $\begin{array}{l}\text { Enfoque: } \\
\text { Cualitativo } \\
\text { Diseño: No } \\
\text { precisa }\end{array}$ & 11 docentes & $\begin{array}{l}\text { Con respecto a la educación continua dentro del alcance de EA para la IE, es } \\
\text { necesario que estas formaciones se piensan desde la singularidad que es actuar } \\
\text { en esta etapa, que requiere algunos detalles. Además, deben adoptar un enfoque } \\
\text { crítico. }\end{array}$ \\
\hline $\begin{array}{l}\text { Enfoque: } \\
\text { Cualitativo } \\
\text { Diseño: Estudio } \\
\text { de casos }\end{array}$ & 15 docentes & $\begin{array}{l}\text { La formación práctica y crítica, basada en la reflexión, la colaboración y el } \\
\text { intercambio, contribuye al cambio, transformación, transiciones ecológicas para los } \\
\text { docentes, teniendo en cuenta que su concepciones y praxis, después de la } \\
\text { participación activa en nuestro proyecto de formación, se hizo más inclusivo, } \\
\text { dialógico, activo, socio-constructivista y democrático. }\end{array}$ \\
\hline
\end{tabular}

Enfoque:

Cualitativo

Diseño: No

10 docentes

precisa

$\begin{array}{lc}\text { Enfoque: } & 9 \text { alfabetizadores } \\ \text { Cualitativo } & 2 \text { directores }\end{array}$

Diseño: Estudio

de casos

Enfoque:

Cualitativo

Diseño: Estudio

14 docentes

de casos
Los resultados evidencian que los maestros reconocen al PIBID como un medio para su educación continua, constituyendo un potencial del programa.

Fuente: Datos obtenidos en la base de datos disponibles en Scielo, Scopus, Ebsco y Dialnet

\section{Discusión}

Luego de aplicar los criterios de selección en el estudio, se identificaron 16 artículos científicos respecto al tema en mención la para la revisión sistemática. Obteniendo como resultados respecto a la cantidad de publicaciones por año que, entre los años 2010 al 2012 no se registraron ninguna publicación, sin embargo, a partir del 2013 la cantidad de publicaciones comenzó a regularse con una tendencia positiva, siendo los años 2018 y 2019 el periodo de tiempo donde se registraron el mayor número de publicaciones. Los primeros trabajos en relación a la temática de estudio, datan de finales del 90 , por lo que es posible verificar que el tema tardo casi dos décadas en concentrar la atención de los investigadores, por lo que podemos deducir que todavía la temática de la formación continua de docentes se encuentra en un estado inicial de desarrollo en estas bases de datos.

En relación a la cantidad de publicaciones por países, se ha considerado el contexto de América Latina, siendo Brasil es el país que ha tenido el mayor número de publicaciones en este estudio, seguido de México y Colombia, sin embargo, no se ha identificado publicaciones de los otros países que conforman América Latina.

La metodología utilizada en los estudios seleccionados, corresponden a los enfoques cualitativo, cuantitativo y mixto (Creswell, 2018). Asimismo, dentro del enfoque cualitativo se han identificado 13 artículos científicos con diseño de investigación acción, estudio de casos y descriptivo, siendo el estudio d casos el diseño de investigación más utilizado por los autores, del mismo modo, utilizaron el enfoque mixto de diseño experimental en menor escala, siendo estos dos enfoques los más utilizados en las investigaciones, por otro lado, dentro del enfoque cuantitativo sólo se identificó un estudio siendo este el enfoque de menor preferencia por los investigadores. También, 5 artículos solo tipifican el estudio sin especificar el 
diseño empleado. En relación a la muestra, en todos los estudios fueron considerados como muestra de investigación docentes de aula, sin embargo, en un número menor de estudios han sido incluidos también gerentes educativos o directores de escuela y coordinadores como parte de la muestra.

Otro punto a descartar, son los principales resultados por los autores de estas investigaciones seleccionadas respecto al tema de la formación continua docente, donde 7 de ellos concluyen que la formación continua es un proceso mediante la cual se actualizan los conocimientos pedagógicos de los docentes para la transformación de sus prácticas en el aula, este hallazgo se asemeja a lo mencionado por (Galazzi, Gomez y Vazquez (2018) así también, como Garcia-Conislla (2020). Jiménez, González y Tornel (2018). Por otro lado, 6 estudios develaron en sus resultados que la formación continua favorece su desarrollo profesional docente ya que mediante la actualización y el perfeccionamiento de su práctica pedagógica consigue la ampliación del ejercicio de sus funciones. Finalmente, 3 estudios señalaron que el desarrollo de los programas formación continua tuvieron un impacto positivo para potenciar las competencias pedagógicas de los docentes, así como desarrollar la cultura investigativa entre los docentes, por lo tanto, deben ser aplicados como política educativa de los estados inferido del análisis (Guadalupe, León, Rodríguez y Vargas, 2017) y (Quiroz, 2015).

Se determina que este estudio presenta cuatro limitaciones: El número de publicaciones analizadas en el presente estudio no representa la totalidad de artículos científicos producidos por autores latinoamericanos e iberoamericanos, solo los publicados en el periodo de tiempo establecido, el idioma y el contexto geográfico. Las bases de datos no permiten el libre acceso a las publicaciones, ya que se encuentran dirigidas a entidades de educación superior previa identificación o claves. Del mismo modo, algunas bases de datos no cuentan con filtros exhaustivos para que la búsqueda de información sea más precisa. También fueron eliminados trabajos porque no correspondían a las carreras profesionales de educación y ciencias sociales, un porcentaje de artículos correspondían al área de salud e ingeniería, esto ha conllevado a la pérdida importante de información sobre la formación continua de los profesores de educación básica infantil.

\section{Conclusiones}

El estudio demuestra que el número de artículos científicos que incluye el tema de la formación continua en docentes de educación básica infantil en América Latina, presenta un carácter ascendente debido a que este tipo de formación fortalece las competencias pedagógicas de los docentes permitiéndoles la transformación de su labor educativa y de esta manera mejorar la enseñanza de los estudiantes, Así También, favorece el desarrollo profesional de los profesores ampliando el ejercicio de sus funciones dentro del contexto educativo, lo que les ha permitido desempeñarse como especialistas, consultores y formadores. Asimismo, las investigaciones manifiestan que los programas de formación continua tienen un impacto positivo en los docentes porque potencian sus capacidades pedagógicas al crear cultura investigativa e innovadora.

En referencia al contexto geográfico, el estudio abarcó América Latina, siendo Brasil el país con el mayor número de publicaciones respecto a la temática de formación continua de docentes en educación básica infantil, así también México presenta un menor número de publicaciones respecto al tema al igual que Colombia. Sin embargo, en los demás países de este contexto no se halló ninguna publicación respecto al tema en el periodo de tiempo previsto de acuerdo a la búsqueda en las diferentes seleccionadas para este estudio.

En cuanto a la metodología, un número significativo de artículos analizados utilizaron el enfoque cualitativo, de diseños descriptivo, estudio de casos e investigación acción, así mismo, utilizaron el enfoque mixto de diseño exploratorio. Sin embargo, sólo se registró un estudio correspondiente al enfoque cuantitativo. En concerniente a la muestra, los estudios abordaron a docentes, sin embargo, algunos de ellos involucraron la participación de directivos, gerentes y coordinadores. Finalmente, los resultados confirmaron que la aplicación de programas de formación continua tuvo efectos positivos en la vicisitud del ejercicio docente y el desarrollo profesional docente.

\section{Fuente de financiamiento}

La presente investigación fue autofinanciada.

\section{Contribución de los autores}

Autor único.

\section{Conflicto de Interés}

Declaramos no tener conflicto de interés.

\section{Referencias bibliográficas}

Aneas Novo, M. C., Sánchez Rodríguez, J., \& Sánchez Rivas, E. (2019). Valoración de la formación del profesorado: comparativa entre autoformación y formación presencial. Gestión Y Análisis De Políticas Públicas, 11(21), 94-108. https://doi.org/10.24965/gapp.v0i21.10569

Ballesteros, M.; Mercado M. y García N. (2019). La formación docente en línea: experiencias con MOOCs en Sonora (México). Realia, 23: 62-79. DOI: 10.7203/realia.23.15903 